\title{
Estimación del forzamiento radiativo debido a aerosoles, vapor de agua y nubes a partir de las medidas del radiómetro multi-filtros con banda de sombra rotante MFRSR en el Observatorio Atmosférico de Izaña entre 2009 y 2011
}

Nota técnica 12 de AEMET

\section{Pedro Miguel Romero Campos ${ }^{(1)}$ Rosa Delia García Cabrera ${ }^{(1),(2)}$ Victoria E. Cachorro Revilla ${ }^{(2)}$}

Enero de 2013 


\section{datos \\ abiertos}

Aviso Legal: los contenidos de esta publicación podrán ser reutilizados, citando la fuente y la fecha, en su caso, de la última actualización

\section{Edita:}

(C) Ministerio de Agricultura, Alimentación y Medio Ambiente Agencia Estatal de Meteorología

Madrid, 2013

Catálogo de Publicaciones de la Administración General del Estado: https://cpage.mpr.gob.es

NIPO: 281-13-005-9

https://doi.org/10.31978/281-13-005-9

Agencia Estatal de Meteorología (AEMET)

C/ Leonardo Prieto Castro, 8

28040 Madrid

http://www.aemet.es/

@Aemet_Esp

https://www.facebook.com/AgenciaEstataldeMeteorologia 


\section{Índice}

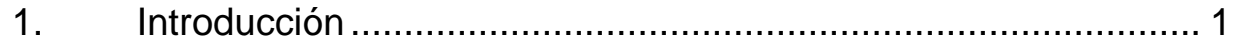

2. Calibración de la irradiancia .................................................... 5

2.1. Identificación de datos afectados por nubes.................. 5

2.2. Calibración en banda ancha ........................................ 7

2.3. Modelización del cociente difusa/global...................... 14

2.4. Comparación radiación BSRN y MFRSR ..................... 18

3. Descripción de los modelos radiativos ................................... 22

3.1. Modelo de Gueymard "ampliado" .............................. 22

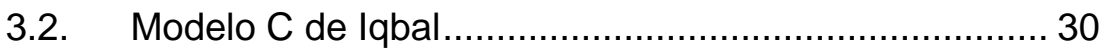

3.3. Modelo SOL - GOA ............................................ 32

3.4. Variables de entrada a los modelos........................... 34

4. Comparativa de los modelos usados ...................................... 36

5. Forzamiento del aerosol, vapor de agua y nubes ................... 40

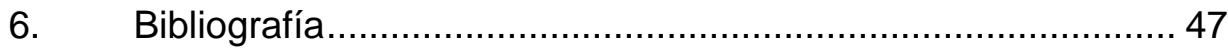





\section{Introducción}

El objetivo de esta nota técnica es ofrecer al lector debidamente formado una metodología de trabajo sencilla que le pueda servir como referencia básica y herramienta introductoria en el estudio de la influencia que un cierto componente atmosférico ejerce sobre la radiación solar que llega a la superficie terrestre. Esta influencia es lo que denominamos forzamiento radiativo. Se ha intentado dar a la exposición un tono pedagógico y didáctico; no obstante, es conveniente poseer ciertos conocimientos básicos en la materia para un mejor aprovechamiento. Aunque los resultados finales obtenidos en este trabajo son considerados como estimaciones orientativas y muy generales, esperamos que la metodología presentada sirva de orientación al futuro investigador que quiera abordar y tratar esta cuestión de una manera más detallada, profunda y precisa.

Uno de los temas clave en el conocimiento del cambio climático consiste en evaluar el impacto cuantitativo y cualitativo que la presencia del vapor de agua, de los aerosoles y de las nubes ejerce en la radiación solar que llega a la superficie terrestre.

El vapor de agua es el gas de mayor efecto invernadero de la atmósfera, y su papel, es crucial como vehículo de intercambio energético en los procesos atmosféricos a través del calor latente de vaporización o condensación. Por otro lado, los aerosoles influyen de forma directa en el balance radiativo terrestre absorbiendo y dispersando la radiación que incide sobre ellos, y de forma indirecta, actuando como núcleos de condensación nubosos sobre los cuales se forman las gotas de agua o cristales de hielo que, si se dan las condiciones necesarias, pueden originar procesos de precipitación. Finalmente, la cobertura nubosa es también un factor muy importante a tener en cuenta en el balance radiativo terrestre, ya que, influye de diversas maneras actuando, bien como escudo ante la radiación que llega, o como tapadera ante la que se refleja y pretende salir hacia el espacio exterior. Además, dependiendo del tipo de nubes, de su ubicación en el cielo y de su espesor, la radiación que incide sobre ellas es dispersada o difundida de formas muy variadas.

El problema de determinar cómo afecta a la radiación que medimos en superficie la presencia de una cierta cantidad de un determinado componente atmosférico es, en general, complicado. No obstante, podemos intentar realizar algún cálculo aproximado o estimación conveniente. Para ello, debemos simular qué radiación recibiríamos y mediríamos con nuestros equipos si dicho componente no estuviera presente en la atmósfera. Esta sería una situación ideal y no real. Aquí es donde entran en juego los denominados modelos de transferencia radiativa. Estos modelos solucionan la ecuación de transferencia radiativa bajo ciertas hipótesis y son capaces de reproducir, con un cierto grado de precisión, tanto la radiación real que medimos con nuestros equipos a partir de los valores de ciertos parámetros que son variables de entrada al modelo, como la radiación solar que mediríamos en situación ideal de ausencia de algún componente determinado. De esta manera, el forzamiento radiativo de un cierto componente sería la diferencia entre la radiación que medimos 
realmente con nuestro equipo, y la que mediríamos en ausencia de dicho componente y que calculamos a través del modelo.

Para obtener el forzamiento radiativo, el primer paso que habría que dar sería tener el equipo de medida muy bien calibrado con respecto al valor de la constante solar que corresponda al rango espectral cubierto por el ancho de banda del canal de medida. El segundo paso, sería elegir un modelo adecuado de radiación solar que combine, en una buena solución de compromiso, la simplicidad con el rigor. Seguidamente, en un tercer paso, deberíamos evaluar cuál es la diferencia entre el valor real medido por el equipo y el calculado por el modelo para el mismo instante de tiempo a partir de los valores reales de las variables de entrada. En este sentido, hay que tener en cuenta que un modelo es tanto más bueno cuanto más se aproxime la predicción que hace de la radiación solar a las medidas reales obtenidas cada día con el equipo. Por último, como cuarto paso, habría que realizar las diferencias entre las medidas reales y las simuladas por el modelo en ausencia de aerosoles, o vapor de agua o nubes.

Son muchos los autores (Bird, (1986); Gueymard, (1998), Casiniere, (1997); Barnard, (2004); Ineichen, (2008), etc.) que han realizado diferentes hipótesis y aproximaciones usando parametrizaciones convenientes de las variables de entrada en diversos modelos, tanto empíricos, como de transferencia radiativa. Una primera aproximación, consiste en usar modelos válidos únicamente para días sin nubes o despejados. Utilizar modelos donde se tienen en cuenta la presencia de nubes es más complicado, y para tales días, no son tan precisos pudiendo conducir a errores difícilmente evaluables.

Un inconveniente importante para desarrollar este tipo de estudios es la alta variabilidad espacio-temporal de los parámetros que han de usarse como variables de entrada y de los que difícilmente disponemos de medidas que sean, al mismo tiempo, suficientemente precisas y de gran resolución temporal. Es necesario asumir ciertos perfiles medios 0 valores climatológicos de determinadas variables de entrada en los modelos y luego caracterizar cómo influye un cambio en dichos parámetros en el forzamiento final calculado, lo que nos daría una idea del error asociado. En este aspecto, nosotros consideramos que todos los errores asociados (error instrumental, errores en las variables de entrada a los modelos, errores en los cálculos debido a las hipótesis o parametrizaciones, etc.) están englobados en la diferencia entre las medidas reales y las suministradas o simuladas por el modelo usando como variables de entrada las obtenidas por otros equipos de alta precisión.

El estudio del forzamiento radiativo que aquí se presenta, se llevará a cabo analizando la radiación solar en banda ancha medida bajo condiciones atmosféricas diversas en el Observatorio Atmosférico de Izaña (OAI, 2370m.s.n.m., Tenerife, España) por el radiómetro de banda de sombra rotante MFRSR (Multifilter Rotating Shadowband Radiometer). Este equipo, que es bastante compacto, completo y manejable (Figura 1.1) toma una medida cada minuto promediada entre 4 tomadas cada 15 segundos y mide, al mismo tiempo, las tres componentes de la radiación solar (global, difusa y directa) en 7 canales: el de banda ancha (similar a los de los piranómetros habituales), y 6 
canales de banda estrecha, comprendidos entre los 10 y $12 \mathrm{~nm}$, y centrados en el visible e infrarrojo cercano. Para más detalles, consultar Romero Campos P.M. et al. (2009).

El radiómetro MFRSR se instaló y comenzó sus medidas en el OAI en febrero de 1996 pero sufrió una avería grave a principios del año 2000 tras la que fue completamente reformado y calibrado trasladándose a otra ubicación provisional durante unos dos años. A principios de 2004, fue nuevamente ubicado en el OAl. Por esta razón, la serie de medidas en Izaña contiene dos períodos claramente diferenciados por un salto y separados por una zona intermedia de ausencia de datos que abarca desde 2000 a 2004.

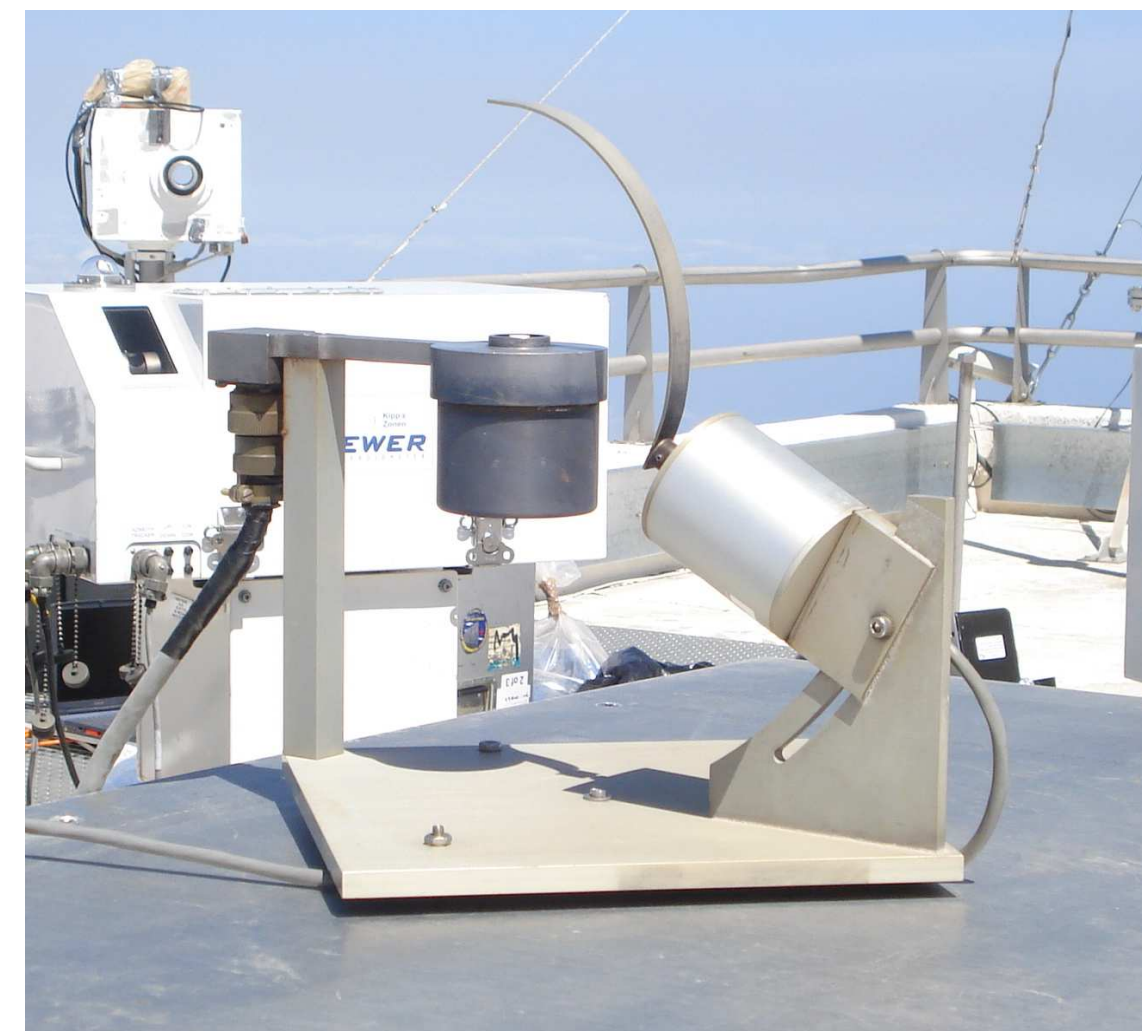

Figura 1.1. Multifilter Rotating Shadowband Radiometer (MFRSR) en el OAI.

Esto supone un grave inconveniente para el estudio preciso que se requiere si quisiéramos abarcar la totalidad del período comprendido entre 1996 y 2011. A esto hay que añadir, que son necesarias medidas de alta calidad, resolución temporal y precisión, tanto de agua precipitable como de espesor óptico de aerosoles, que sirvan de entrada a los modelos que usaremos.

Los equipos más precisos de los que disponemos de tales medidas son, por un lado y en relación con el espesor óptico de aerosoles, el Radiómetro de Filtros de Precisión (PFR, Precision Filter Radiometer) que lleva midiendo en el OAl desde junio de 2001 y, por otro lado y en relación con la medida de agua precipitable en columna, los tradicionales sondeos PTU con medidas desde 1995 realizadas con sondas RS80/RS92, y un receptor GPS-GLONASS, perteneciente al Instituto Geográfico Nacional (IGN), que comenzó a medir en el OAI en julio de 2008. 
El PFR forma parte de una red de fotómetros similares repartidos por todo el mundo dentro del programa de Vigilancia Atmosférica Mundial (VAG) y pertenece al Centro Mundial de Radiación (WRC, World Radiation Center, de Davos (Suiza)). El equipo mide radiación directa normal cada minuto en cuatro canales de banda estrecha centrados en $368 \mathrm{~nm}, 412 \mathrm{~nm}, 500 \mathrm{~nm}$ y $862 \mathrm{~nm}$ para la obtención precisa del espesor óptico de aerosoles.

Las medidas de agua precipitable integradas en la columna atmosférica son obtenidas dentro del Programa de Radiación, Vapor de Agua y Aerosoles en Columna del OAI a partir de los sondeos PTU diarios, cuya resolución temporal aporta sólo dos valores al día (a las Oh UTC y 12h UTC) y, también, con resolución temporal de 15 minutos o de 1 hora, a partir de los retrasos cenitales totales (ZTD, Zenith, Total Delay) de la señal GPS-GLONASS recibida en nuestro receptor a su paso por la atmósfera. La resolución temporal va asociada al tipo de órbita GPS (ultrarrápida ó precisa para 15 minutos ó 1 hora, respectivamente).

Para más detalles sobre toda la instrumentación aquí presentada y el procedimiento de obtención de los datos consultar en Romero Campos P.M. et al. (2009).

Como se desprende de todo lo que acabamos de decir, el período de solape de los datos de mejor calidad y con más resolución temporal de todos los equipos tendría que ser a partir de julio de 2008. Por todo ello, descartaremos en nuestro estudio el período previo a la avería del MFRSR así como el período de ausencia de datos posterior; teniendo en cuenta, además, que desde enero de 2009 calculamos agua precipitable GPS a partir de órbitas precisas (no sólo las ultrarrápidas), se decidió restringir el período de estudio del forzamiento radiativo al comprendido desde 2009 a 2011.

En los capítulos siguientes, iremos exponiendo la metodología seguida para la obtención del forzamiento radiativo de aerosoles, vapor de agua y nubes consistente en los cuatro pasos descritos al comienzo de este capítulo.

Propondremos también un método sencillo que nos puede ayudar a estimar, para la atmósfera real, la irradiancia difusa y global horizontales a partir de un modelo inicialmente concebido para la evaluación de la irradiancia directa normal en banda ancha.

Finalmente, evaluaremos el alcance y validez de todas nuestras estimaciones. 


\section{Calibración de la irradiancia}

En este capítulo exponemos, de forma sistemática, el procedimiento empleado para obtener una constante o factor de calibración que nos permita escalar adecuadamente los valores de la irradiancia solar medidos por nuestro radiómetro MFRSR en el mismo rango espectral en el que miden los piranómetros de la Red de Referencia Mundial para la medida de la Radiación en Superficie (red BSRN) instalados en el OAI. También modelizaremos el cociente entre la irradiancia difusa y la global horizontales por ser una variable de interés en la identificación de datos afectados por nubes. Finalmente, haremos una comparación entre los valores de la serie de radiación total diaria calibrada en banda ancha del radiómetro MFRSR con los correspondientes de la BSRN en el período de estudio a fin de validar tanto el procedimiento empleado como el factor de calibración obtenido.

\subsection{Identificación de datos afectados por nubes}

El primer paso que daremos será el de caracterizar y etiquetar cada dato de irradiancia medido por nuestro radiómetro mediante un código numérico o flag que nos dé información sobre si el dato es o no válido, y en caso de que sea válido, si está o no afectado por nubes. El criterio que seguiremos para asignar un valor a este código será el siguiente:

- Dato válido y no afectado por nubes: flag $=0$.

- Dato válido y afectado por nubes: flag $=1$.

- Dato no válido para nuestro estudio: flag $=\mathbf{- 1}$.

Es necesario realizar esta caracterización inicial, ya que, el efecto de las nubes en la radiación solar se obtendrá restándole a la irradiancia real medida en un dato nuboso una estimación de la que le correspondería, ese mismo día e instante, simulada con un modelo válido para días despejados. Por otro lado, hay que descartar los datos que corresponden a ruido instrumental y también los que están afectados por efectos ajenos al proceso de medida y que los hacen inviables para el estudio.

La herramienta que usaremos para etiquetar los datos está basada en la modelización del cociente entre la irradiancia difusa (D) y la irradiancia global (G), ambas en banda ancha y medidas en un plano horizontal bajo un ángulo sólido de $2 \pi$. Long y Ackerman, (2000) denominan a este cociente "diffuse ratio". Nosotros lo llamaremos cociente o proporción difusa/global y lo denotaremos como IC:

$$
I_{C}=\frac{D}{G}
$$

Para un día despejado (sin nubes) y limpio (escasa presencia de aerosoles), la radiación difusa suele ser un 10\% de la global (Iqbal M., 1983). En el caso de Izaña, es incluso inferior tomando valores frecuentes en torno al $5 \%$. Para tales días, el cociente difusa/global valdría entre $[0.05,0.1]$, 
aproximadamente. Para un día despejado, pero no suficientemente limpio, la radiación difusa es superior al $10 \%$ de la global y podría alcanzar incluso el $20 \%$. Consideraremos pues, que el cociente difusa/global en días despejados debería ser inferior o igual a 0.2. Por otro lado, entre las componentes de la irradiancia global horizontal (G), la irradiancia difusa horizontal (D), y la irradiancia directa horizontal $(R)$ siempre se cumple la siguiente relación:

$$
G=D+R \quad(2.1 .2)
$$

De esta manera, para un día completamente cubierto por nubes, el cociente difusa/global valdría teóricamente 1 porque la radiación directa sería nula y la global sería únicamente radiación difusa.

Tal y como proponen Long y Ackerman, (2000), la proporción difusa/global, para el caso de días limpios y despejados, puede ser modelizada por una función potencial simple del coseno del ángulo cenital solar. Si llamamos $\mu_{0}$ a este coseno, resulta que la proporción difusa/global se puede expresar como:

$$
I_{C}=a \mu_{0}^{b} \quad(2.1 .3)
$$

Para cada día, se puede hacer un ajuste robusto por la técnica de mínimos cuadrados a dicha función potencial lo que nos proporcionará un valor diario adecuado de los coeficientes $a$ y $b$. Este ajuste se realizará en cuatro fases. En la primera fase, descartaremos aquellas medidas tomadas en instantes de tiempo para los cuales los valores de irradiancia directa de alguno de los canales de banda estrecha de nuestro radiómetro no superen un umbral de ruido instrumental de $0.05 \frac{W}{\mathrm{~m}^{2} \mathrm{~nm}}$, y también, aquellas para las que el valor de irradiancia directa normal en banda ancha sea menor o igual a $50 \frac{\mathrm{W}}{\mathrm{m}^{2}}$. El día será descartado de nuestro estudio si no podemos encontrar al menos 10 valores que cumplan con esta primera condición.

En una segunda fase, realizamos sobre la selección de valores anteriores, un primer ajuste a nuestra función potencial descartando aquellos que se alejen de dicho ajuste al menos 3 veces el valor del error cuadrático medio respecto del ajuste. Esta última cantidad viene dada por:

$$
R c m=\sqrt{\frac{\sum\left(D / G-a \mu_{0}^{b}\right)^{2}}{N-2}}
$$

Donde $\mathrm{N}-2$ es el número de valores usados en el ajuste menos el ํํ de grados de libertad del mismo.

De nuevo, si no hay al menos 10 valores que pasen este segundo filtro, el día es descartado. 
En una tercera fase, con los valores que han superado el segundo filtro, realizamos un nuevo ajuste. A partir de aquí, tomaremos como dato no nuboso aquél cuyo cociente difusa/global sea igual o inferior a 0.2 y cuya distancia al ajuste final sea inferior a 3 veces el error cuadrático medio de este segundo ajuste. Como dato nuboso tomaremos aquél que iguale o sobrepase esta última cantidad o que su cociente difusa/global sea superior a 0.2 , o ambas cosas. Finalmente, los datos no válidos son los que no pasaron el primer filtro, y también, aquellos en los que el cociente difusa/global fuese inferior o igual a 0.

Por último, en la cuarta fase, escogeremos únicamente los datos no afectados por nubes y obtendremos, a partir de ellos y haciendo un ajuste final, los parámetros $a$ y $b$ así como el error cometido en su cálculo por el empleo de la técnica de mínimos cuadrados. El error cometido lo expresaremos en \% respecto del valor de la pendiente del ajuste.

Con objeto de evitar tener que realizar dos ajustes por día debido a la simetría de la radiación antes y después del mediodía solar, y también para dar más estabilidad a los propios ajustes, se ha procedido a realizarlos de la forma que se expone a continuación.

Tomando logaritmos neperianos en ambos miembros de la igualdad (2.1.3), se tiene que:

$$
\ln \left(I_{C}\right)=\ln (a)+b \ln \left(\mu_{0}\right)
$$

Y dividiendo cada factor de (2.1.5) por el $\ln \left(\mu_{0}\right)$, resulta que:

$$
y=\frac{\ln \left(I_{C}\right)}{\ln \left(\mu_{0}\right)}=\frac{1}{\ln \left(\mu_{0}\right)} \ln (a)+b=A x+b
$$

Esta expresión no es otra cosa que la ecuación de una recta de pendiente $\ln (a)$ y de ordenada en el origen $b$, tomándose como variable independiente $x=\frac{1}{\ln \left(\mu_{0}\right)}$ y como variable dependiente $y=\frac{\ln \left(I_{C}\right)}{\ln \left(\mu_{0}\right)}$.

\subsection{Calibración en banda ancha}

Sabemos que la ley de Bouguer-Lambert-Beer, nos da una relación entre la irradiancia recibida, normal a la dirección de incidencia en un punto de la superficie terrestre $I_{\lambda}$, y la irradiancia que incide en la parte superior de la atmósfera $I_{0 \lambda}$ (irradiancia extraterrestre).

$$
I_{\lambda}=I_{0 \lambda} \times e^{-m \tau(\lambda)}
$$


Los diversos componentes atmosféricos ejercen diferentes atenuaciones sobre la irradiancia extraterrestre a medida que esta atraviesa la atmósfera. La atenuación varía en función de la longitud de onda $\lambda$ y viene expresada por el producto entre la masa óptica relativa a la dirección cenital, $m, \mathrm{y}$ el coeficiente de extinción por unidad de masa óptica, $\tau(\lambda)$. Rigurosamente hablando, la ley (2.2.1) sólo se cumple para banda estrecha, es decir, para radiación que puede ser considerada como monocromática por ser muy pequeño (del orden de 10$12 \mathrm{~nm}$, o inferiores) el ancho de banda del canal de medida en torno a un valor nominal central. No obstante, haciendo una integración espectral de la ley (2.2.1) podemos establecer, en primera aproximación, su validez para un espectro de banda ancha. En el caso que nos ocupa, el ancho de banda donde existe una eficiencia del $95 \%$ de detección para los piranómetros usados por la Red de Referencia Mundial para la medida de la Radiación en Superficie, red BSRN (Baseline Surface Radiation Network), está comprendido entre los $330 \mathrm{~nm}$ y los $2600 \mathrm{~nm}$. Este será el ancho de banda patrón que elegiremos a fin de hacer comparables los datos de nuestro radiómetro MFRSR con los de la BSRN. El OAl pertenece a la red BSRN desde marzo de 2009 (www.aemet.izana.org/bsrn_iza).

Dado que el ancho de banda espectral del canal "broadband" del MFRSR está comprendido entre los $300 \mathrm{~nm}$ y los $1100 \mathrm{~nm}$, será necesario escalarlo adecuadamente. Esto lo conseguiremos calibrando el instrumento frente a la constante solar o irradiancia extraterrestre correspondiente al rango [330 $\mathrm{nm}$, $2600 \mathrm{~nm}$ ] medida a la distancia Tierra-Sol de una unidad astronómica (1 UA).

El valor de la constante solar aceptado y recomendado por la Organización Meteorológica Mundial (OMM) para todo el espectro solar a la distancia de 1 $U A$, es de $I_{0}=1367 \frac{\mathrm{W}}{\mathrm{m}^{2}}$. En nuestro caso, el valor de dicha constante será menor puesto que no consideramos todo el espectro solar sino sólo el intervalo [330nm, 2600nm]. Para obtener el valor adecuado de la constante solar en este intervalo, usaremos el espectro solar extraterrestre proporcionado por Kurucz, (1992), el cual, ha sido promediado en intervalos de $1 \mathrm{~nm}$ alrededor de las diferentes longitudes de onda nominales comprendidas en el intervalo [250nm, $10000 \mathrm{~nm}]$. Empleando una integración numérica por la regla de los trapecios aplicada al intervalo [330 $\mathrm{nm}, 2600 \mathrm{~nm}$ ] obtenemos, para la constante solar a 1 UA, el siguiente valor:

$$
I_{0}=1288 \frac{W}{m^{2}}
$$

Por otro lado, existen diversas aproximaciones para obtener un valor adecuado, en banda ancha, de la masa óptica relativa a la dirección cenital. Nosotros usaremos la expresión (2.2.3) recomendada y utilizada en el modelo r.sun del Instituto para la Energía y el Transporte (IET) del Centro de Investigación Conjunta (JRC, Joint Research Centre) de la Comisión Europea. El modelo de radiación solar r.sun está implementado en el sistema de información geográfica GRASS GIS y está escrito en lenguaje C. Es de código 
fuente abierto y libremente disponible en su versión 5.0.0. Para más información consultar en:

http://re.jrc.ec.europa.eu/pvgis/solres/solmod3.htm\#clear-sky radiation

También, las correcciones por refracción atmosférica y por la altitud de la estación, así como la expresión para la excentricidad terrestre son las propuestas por el IET y usadas en r.sun.

$$
m=\frac{\exp \left(\frac{-z}{8434.5}\right)}{\sin \left(h_{0}^{\text {ref }}\right)+0.50572 \times\left(h_{0}^{\text {ref }}+6.07995\right)^{-1.6364}}
$$

Aquí, $z$ es la altitud de la estación en m.s.n.m. y $h_{0}^{\text {ref }}$ es la elevación solar, expresada en grados y corregida por refracción atmosférica. Si llamamos $h_{0}$ a la elevación solar sin corregir por refracción tenemos que:

$$
h_{0}^{r e f}=h_{0}+\frac{0.061359 \times\left(0.1594+1.123 \times h_{0}+0.065656 \times h_{0}^{2}\right)}{1+28.9344 \times h_{0}+277.3971 \times h_{0}^{2}}
$$

La expresión de la irradiancia normal $I$ a la dirección de incidencia para banda ancha a la distancia Tierra-Sol de 1 UA podría expresarse como:

$$
I=I_{0} \times \operatorname{exc} \times \exp \left(-m \times\left(R a y+\sum_{i} \mathrm{~T}_{i}\right)\right)
$$

Donde $e x c=1+0.03344 \times \cos \left(\frac{2 \pi j}{365.25}-0.048869\right)$ es la excentricidad de la órbita terrestre, $j$ es el día natural del año (de 1 a 365 (366)), $T_{i}$ es la atenuación de la atmósfera para el componente $i$ (básicamente, vapor de agua y aerosoles) y Ray es la atenuación por dispersión Rayleigh debida a las moléculas de aire. Esta última viene dada para $m \leq 20$ por:

$$
\text { Ray }=\frac{1}{6.6296+1.7513 \times m-0.1202 \times m^{2}+0.0065 \times m^{3}-0.00013 \times m^{4}}
$$

Y para $m>20$ por:

$$
\text { Ray }=\frac{1}{10.4+0.718 \times m}
$$

Una forma más útil y conveniente de expresar la anterior relación 2.2.5 es introduciendo el denominado factor de turbiedad de Linke, $T_{L}$ que se define como el número de atmósferas limpias y secas (sólo dispersión Rayleigh) 
necesarias para igualar la atenuación y la irradiancia a los valores reales. De esta forma, la expresión 2.2.5 quedaría así:

$$
I=I_{0} \times \operatorname{exc} \times \exp \left(-m \times R a y \times T_{L}\right)
$$

Para eliminar la dependencia de la atenuación por aerosoles o turbiedad con las variaciones de la masa óptica, se toma una masa óptica de referencia igual a 2 a la que Kasten, en 1996, corrigió mediante un factor de 0.8662 , de manera que, la expresión final para 2.2.5, sería la siguiente:

$$
I=I_{0} \times \operatorname{exc} \times \exp \left(-0.8662 \times m \times R a y \times T_{L}\right)
$$

La expresión 2.2.6 es, en realidad, un ajuste conveniente para poder utilizar la ley de Bouguer-Lambert-Beer en banda ancha. Esta será la ecuación base que usaremos para calibrar nuestro equipo.

Es necesario advertir que, aunque la expresión 2.2.6 sea la utilizada por el modelo r.sun, nosotros a la hora de calibrar y escalar adecuadamente el canal de banda ancha del MFRSR, no vamos a realizar ninguna suposición sobre los valores de entrada de la variable $T_{L}$, ni sobre la atenuación o extinción por vapor de agua o por aerosoles. Es decir, no vamos a usar la salida del modelo r.sun para los diferentes días ni vamos a calibrar nuestro equipo frente a ningún otro modelo de radiación. La calibración se realizará frente a la constante solar en 330nm-2600nm usando la expresión 2.2.6 en su forma neperiana y ajustando por mínimos cuadrados.

Tomando logaritmos neperianos en 2.2.6, tenemos que:

$$
\ln (I / \text { exc })=\ln \left(I_{0}\right)-0.8662 \times m \times R a y \times T_{L}
$$

Y dividiendo por $0.8662 \times m \times$ Ray ambos miembros:

$$
\frac{\ln (I / \text { exc })}{0.8662 \times m \times R a y}=\frac{\ln \left(I_{0}\right)}{0.8662 \times m \times R a y}-T_{L}=Y_{C}=X_{C} \times \ln \left(I_{0}\right)-T_{L}
$$

Donde $X_{C}=\frac{1}{0.8662 \times m \times R a y}$ e $Y_{C}=\frac{\ln (I / \text { exc })}{0.8662 \times m \times R a y}$

El ajuste por mínimos cuadrados de $Y_{C}$ frente a $X_{C}$ será la ecuación de una recta de pendiente $\ln \left(I_{0}\right)$. Evidentemente, al realizar el ajuste por mínimos cuadrados la pendiente de dicha recta no nos saldrá exactamente $\ln \left(I_{0}\right)$, sino que se podrá expresar como $\ln \left(I_{0}^{\prime}\right)$. El factor de calibración vendrá entonces dado por $f c a=\frac{I_{0}}{I_{0}^{\prime}}$. 
Para cada día, realizamos este ajuste con aquellos datos que no están afectados por nubes, es decir, de código o flag=0. Si no hay al menos 10 valores que cumplan con esta condición, el día es descartado. Haremos un único ajuste para el día completo con objeto de obtener una constante de calibración que se adapte bien tanto para la mañana como para la tarde. A diferencia del ajuste tradicional, en el que hubiésemos elegido como variable independiente $m$ y como variable dependiente el $\ln (I /$ exc $)$ esta nueva forma de ajustar propuesta da más estabilidad frente a la variación de la masa óptica y menos error en la estimación de la irradiancia extraterrestre del radiómetro por el procedimiento de mínimos cuadrados. En este procedimiento, el error en la pendiente es generalmente menor que el error en la ordenada en el origen. De esta forma, al obtenerse la irradiancia extraterrestre como pendiente, y no como ordenada en el origen, el error asociado es inferior al que hubiésemos obtenido en ese caso.

Realizados los ajustes, obtenemos una serie en el tiempo con un valor dado del factor o constante de calibración para cada día. En la figura 2.2.1 representamos esta serie. En ella, podemos observar cómo muchos de los valores de las constantes de calibración se concentran en una parte central bien definida y destacada respecto de los valores que se alejan de ella. Estos valores, compactados y concentrados, corresponden a días en los que la estabilidad y las condiciones atmosféricas nos han permitido realizar mejores ajustes y calibraciones. Por ello, están relativamente próximos unos a otros.

Calibración en banda ancha del MFRSR

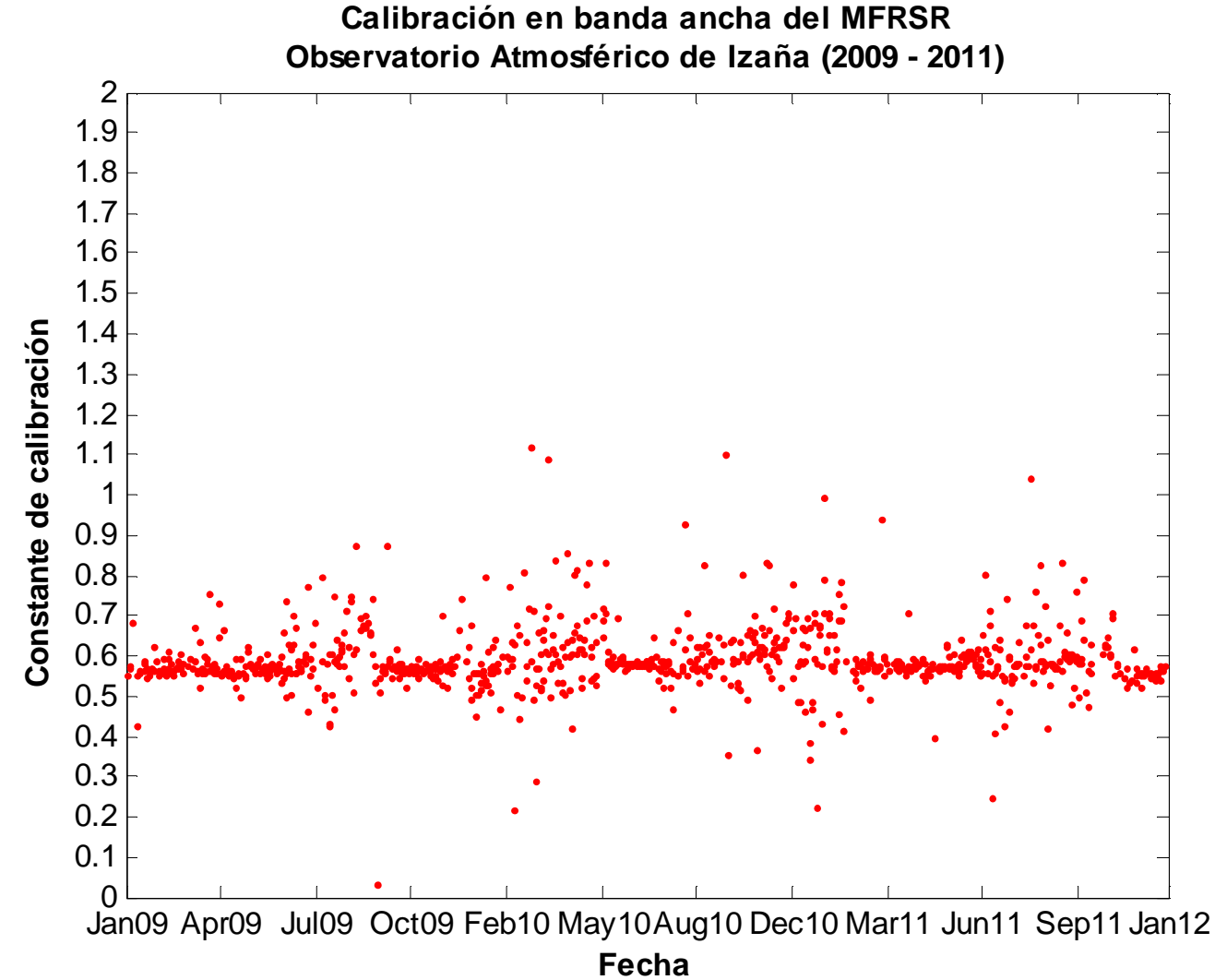

Figura 2.2.1. Constante de calibración del MFRSR en banda ancha. La constante se aplica a las tres componentes de la irradiancia y representa el factor (adimensional) por el que hay que multiplicar a los valores de irradiancia para que la directa normal a la incidencia, en la parte superior de la atmósfera, sea de $1288 \mathrm{~W} / \mathrm{m}^{2}$. 
No obstante, para garantizar una buena calibración y poder estimar con precisión el forzamiento radiativo, se hace necesario elegir o seleccionar, dentro de esta zona central, únicamente aquellos días donde las condiciones atmosféricas y de calibración han sido realmente óptimas.

Para ello, nos basamos en los valores de ciertos parámetros deducidos de los ajustes. Estos valores tendrán que tener en cuenta tanto la calibración diaria como el ajuste del cociente difusa/global diario, y son los siguientes:

- error (\%) en el procedimiento de ajuste por mínimos cuadrados,

- error cuadrático medio respecto del ajuste,

- coeficiente de determinación (correlación al cuadrado)

- número de valores usados en los ajustes.

La selección de los días óptimos de la serie se lleva a cabo eligiendo, convenientemente, los límites máximos y mínimos razonables para cada uno de los parámetros anteriores, según el caso. Es evidente que las calibraciones y los días seleccionados serán tanto mejores y más apropiados cuanto menores sean los dos primeros parámetros y mayores los dos segundos. Ahora bien, las posibles combinaciones de estos 4 parámetros dentro de límites razonablemente buenos son muchas y es normal que, aún así, las constantes de calibración de los días escogidos como óptimos, puedan presentar entre ellas una dispersión significativa. El criterio a seguir entonces sería trocear la serie en intervalos más pequeños tales que los valores de calibración, además de estar dentro de los límites establecidos para los 4 parámetros anteriores, tengan poca dispersión entre sí. Lo ideal, es que la dispersión de las constantes de calibración así elegidas no sobrepase el $1 \%$ del valor promedio de las mismas en el intervalo considerado. Este valor promedio es el que tomamos como factor de calibración en el intervalo y tendría un error asociado equivalente a la desviación estándar respecto de dicho valor medio.

Para los ajustes en días óptimos establecemos, como valores límites o umbrales de los parámetros anteriores, los siguientes:

- errores en los ajustes por mínimos cuadrados: menores del $1 \%$

- errores cuadráticos medios respecto de ajustes: menores de 1

- coeficientes de determinación: mayores de 0.99

- número de valores usados en los ajustes: al menos 100

Se entiende que estos umbrales se aplican tanto en los ajustes para la calibración del canal de banda ancha frente a la constante solar como a los ajustes del cociente difusa/global.

Para asegurar que los valores de calibración tuviesen poca dispersión lo que hicimos fue seleccionar, primeramente, aquellos valores de la zona central cuya dispersión quedaba dentro del rango semi-intercuartílico. Luego, de entre estos últimos, nos quedamos únicamente con aquellos que, además, cumplían con los criterios anteriores. 
En la figura 2.2.2 presentamos los valores iniciales y los seleccionados finalmente según todos estos criterios. A la vista de esta gráfica, no juzgamos necesario trocear en intervalos más pequeños la serie de los valores seleccionados, ya que, estos se distribuyen de una manera razonablemente uniforme a lo largo de toda la serie, ocupando la zona más central de la misma y presentando una dispersión pequeña. El valor promedio de los mismos puede tomarse como valor de calibración y su desviación estándar como error de calibración. Los resultados numéricos asociados se muestran en la tabla 2.2.1. En ella, se puede apreciar que el error de calibración obtenido es inferior al $2 \%$.

Teniendo en cuenta que el propio error instrumental de los piranómetros de la BSRN (que son de la más alta calidad) ya se mueve en torno al $2 \%$ del valor de radiación diario, y que la estabilidad obtenida por García R. D., (2011) en la irradiancia global medida con ellos en el OAI usando el modelo LibRadTran como herramienta de chequeo en el período 2009 - 2010 era del orden del 3$4 \%$, el resultado de nuestra calibración es sin duda un buen resultado.

\begin{tabular}{|c|c|}
\hline \multicolumn{2}{|c|}{ Parámetro } \\
\hline $\begin{array}{c}\text { Constante de calibración } \\
\text { (valor medio) }\end{array}$ & 0.573562 \\
\hline $\begin{array}{c}\text { Desviación estándar } \\
\text { absoluta }\end{array}$ & 0.010509 \\
\hline $\begin{array}{c}\text { Desviación estándar } \\
(\%)\end{array}$ & 1.832209 \\
\hline
\end{tabular}

Tabla 2.2.1. Constante o factor de calibración del MFRSR en banda ancha y parámetros de error asociados. Este factor es adimensional. Por él hay que multiplicar las tres componentes de la radiación MFRSR para escalarlas convenientemente al rango espectral [330nm. 2600nm].

El error en la calibración ya tiene en cuenta al propio error instrumental del equipo y a los relacionados con los procesos de cálculo astronómicos.

Añadir, finalmente, que ya que tenemos un único sensor en el radiómetro que mide simultáneamente las tres componentes de la irradiancia (global, difusa y directa), el mismo factor de calibración obtenido se aplica a cada una de ellas. La calibración consiste en multiplicar esta constante o factor de calibración por el valor de la irradiancia medida. Con ello, conseguimos escalar adecuadamente el canal de banda ancha del MFRSR al rango espectral [330nm, 2600nm].

Desde la expresión (2.1.2) puede verse, matemáticamente, que calibrar la irradiancia directa supone calibrar, con la misma constante, las otras dos componentes:

$$
\begin{gathered}
R=I \times \mu_{0}=G-D \\
R_{c}=f c a \times R=f c a \times I \times \mu_{0}=f c a \times(G-D)=f c a \times G-f c a \times D=G_{c}-D_{c}
\end{gathered}
$$




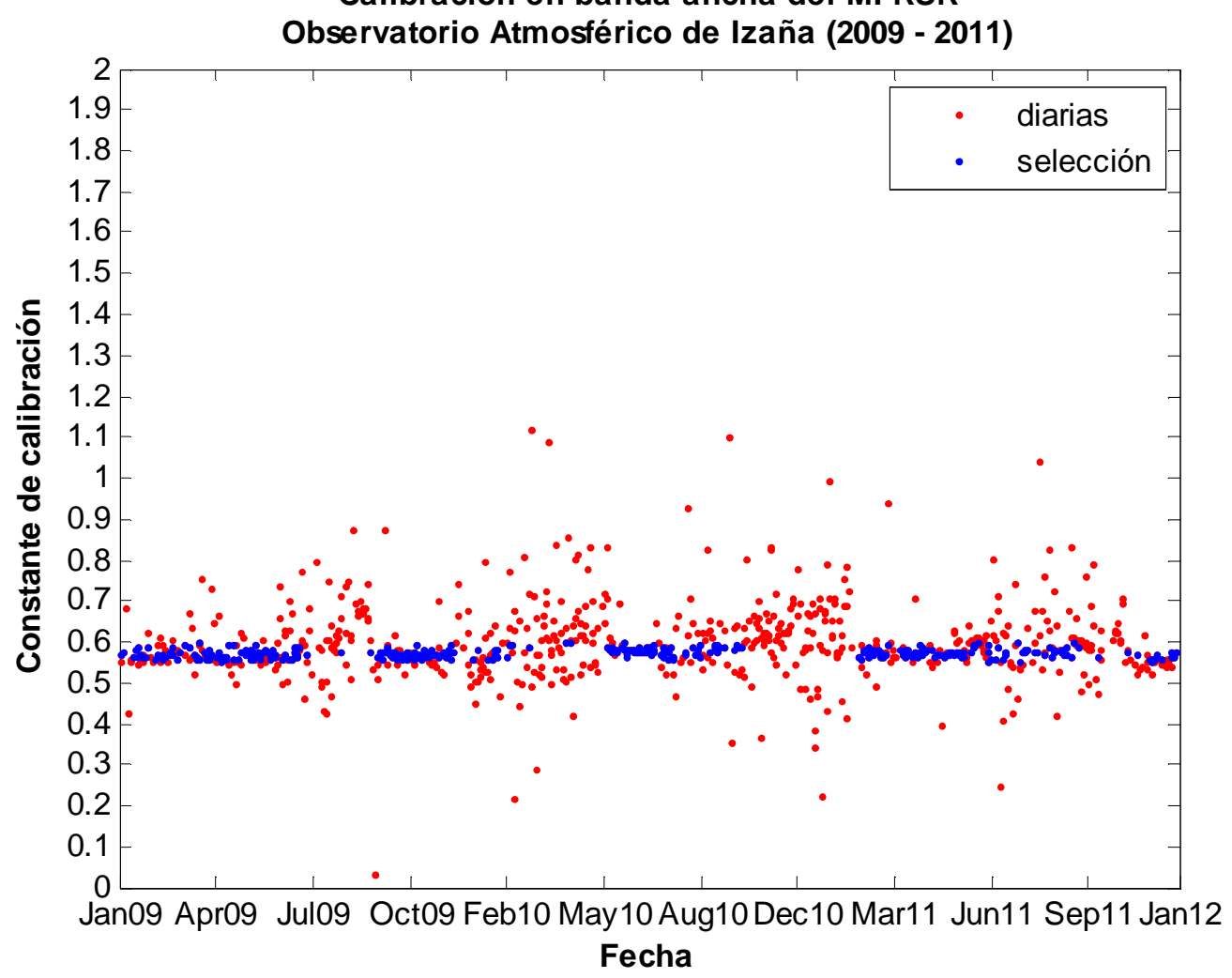

Figura 2.2.2. Selección de las constantes de calibración del MFRSR en banda ancha. Los días seleccionados (azul) corresponden a aquellos cuya calibración y ajuste de cocientes difusa/global diarios cumplen con los requisitos de calidad establecidos por medio de los umbrales. La dispersión entre ellos es inferior al $2 \%$ y ocupan la parte central de la serie.

\subsection{Modelización del cociente difusa/global}

Decíamos que la proporción o cociente difusa/global se podía modelar, para cielos limpios y despejados, mediante una función potencial simple del coseno del ángulo cenital solar (fórmula 2.1.3).

De manera similar a lo realizado en la sección anterior, podemos intentar calibrar las constantes asociadas a la función potencial simple de la fórmula 2.1.3 con el objeto de ver si puede asumirse también un único valor de estas constantes para todo el período.

Seleccionando los mismos días que se utilizaron en la calibración frente al modelo y aplicando el método de mínimos cuadrados descrito en la sección 2.1, obtenemos para $a$ y $b$, los siguientes valores:

\begin{tabular}{|c|c|c|}
\hline Parámetro & $\mathrm{a}$ & $\mathrm{b}$ \\
\hline Valor medio & 0.051678 & -0.709114 \\
\hline $\begin{array}{c}\text { Desviación estándar } \\
\text { Absoluta }\end{array}$ & 0.015966 & 0.144992 \\
\hline $\begin{array}{c}\text { Desviación estándar } \\
(\%)\end{array}$ & 30.894886 & 20.446954 \\
\hline
\end{tabular}

Tabla 2.3.1. Parámetros asociados al cociente difusa/global del MFRSR para el período 2009-2011. 
Como vemos en la tabla, la desviación estándar es alta para ambos parámetros.

Si representamos el máximo cociente difusa/global diario frente a cada uno de ellos para los días seleccionados en la calibración, vemos los resultados que se muestran en las gráficas de la figura 2.3.1.

En estas figuras, se aprecia que, por debajo de un cociente difusa/global máximo diario de 0.19 , aproximadamente, el valor de cada uno de los dos parámetros oscila mucho menos en torno a un valor central que cuando el cociente difusa/global rebasa la cota de 0.19 .

Esta cota puede marcar el grado de limpieza del aire necesario para poder asumir un modelo potencial del cociente difusa/global adecuado.

Para valores por debajo de 0.19 los valores medios y desviaciones estándares de los parámetros del ajuste, en los días de calibración, son los siguientes:

\begin{tabular}{|c|c|c|}
\hline Parámetro & $\mathrm{a}$ & $\mathrm{b}$ \\
\hline Valor medio & 0.042542 & -0.628158 \\
\hline $\begin{array}{c}\text { Desviación estándar } \\
\text { Absoluta }\end{array}$ & 0.004795 & 0.146170 \\
\hline $\begin{array}{c}\text { Desviación estándar } \\
(\%)\end{array}$ & 11.271095 & 23.269627 \\
\hline
\end{tabular}

Tabla 2.3.2. Parámetros asociados al cociente difusa/global del MFRSR en días en los que el cociente difusa/global máximo diario es inferior a 0.19 dentro del período 2009-2011.

De la tabla 2.3.2 se desprende que, respecto de la situación anterior, se ha reducido ostensiblemente el error del coeficiente del ajuste pero ha aumentado ligeramente el del exponente del mismo.

Podríamos concluir que un modelo para Izaña del cociente difusa/global basado en una función potencial simple sería válido para días en los que el cociente difusa/global no supere el valor de 0.19 y tendría una posible variación del coeficiente del modelo en torno al $11 \%$ y del exponente en torno al $23 \%$.

Teniendo en cuenta estas limitaciones, la consideración del modelo para el cociente difusa/global nos puede ayudar en ciertos casos a corregir o ajustar la irradiancia difusa evaluada por los modelos radiativos habituales cuando no se ajusta suficientemente a la realidad. También nos puede servir para completar aquellos otros modelos parciales en los que sólo se modeliza la irradiancia directa normal. Como veremos en el capítulo siguiente, este es el caso del modelo de Gueymard.

Si únicamente tenemos modelizada la irradiancia directa normal $R_{n m}$, a partir de esta, se puede obtener la irradiancia directa horizontal multiplicando por el coseno del ángulo cenital solar, de forma que, 


$$
R_{h m}=R_{n m} \times \mu_{0}
$$

Si llamamos $D_{h m}$ y $G_{h m}$ a las irradiancias difusa y global horizontales cuya modelización queremos obtener, y $D_{C}$ y $G_{C}$ a las medidas por el instrumento, y asumimos que el cociente difusa/global del modelo sea igual al del instrumento, resulta que, dividiendo por $G_{h m}$ ambos miembros de la expresión 2.1.2 particularizada al modelo, obtenemos:

$$
1=\frac{D_{h m}}{G_{h m}}+\frac{R_{h m}}{G_{h m}}
$$

Como exigimos que:

$$
\frac{D_{C}}{G_{C}}=\frac{D_{h m}}{G_{h m}}
$$

Substituyendo en 2.3.1, obtenemos para $G_{h m}$ y $D_{h m}$ :

$$
G_{h m}=\frac{R_{h m}}{1-\frac{D_{C}}{G_{C}}} \text { (2.3.3) y } D_{h m}=\frac{R_{h m}}{1-\frac{D_{C}}{G_{C}}} \times \frac{D_{C}}{G_{C}}
$$

O bien, asumiendo el modelo para el cociente difusa/global basado en una función potencial simple, tendremos:

$$
G_{h m}=\frac{R_{h m}}{1-a \mu_{0}^{b}} \quad \text { (2.3.3) y } D_{h m}=\frac{R_{h m}}{1-a \mu_{0}^{b}} \times a \mu_{0}^{b}
$$

De esta forma, hemos conseguido modelizar y estimar, a partir de la irradiancia directa normal las otras dos componentes de la irradiancia (global y difusa). La hipótesis básica de asumir para el modelo el mismo cociente difusa/global que mide el instrumento es razonable si el equipo está correctamente calibrado y mide bien. En este caso, el modelo será tanto mejor cuanto más se ajuste a dichas medidas. Sin embargo, el problema de modelizar basándonos exclusivamente en un ajuste experimental de datos de un radiómetro bien calibrado, y dejando aparte consideraciones de tipo físicoatmosférico, no nos permitiría estimar las componentes del modelo para una atmósfera ideal sin aerosoles o sin vapor de agua ya que no conocemos la respuesta instrumental en este caso. Por esta razón, cuando hablemos del modelo de Gueymard en el capítulo siguiente, explicaremos cómo haremos la "ampliación" a las componentes global y difusa para el caso de una atmósfera ideal en ausencia de aerosoles o de vapor de agua precipitable.

El cociente difusa/global experimental lo usaremos como herramienta de detección e identificación de datos nubosos y, también, en la evaluación de las componentes global y difusa de la radiación para una atmósfera real. 

MFRSR. OAI. 2009 - 2011

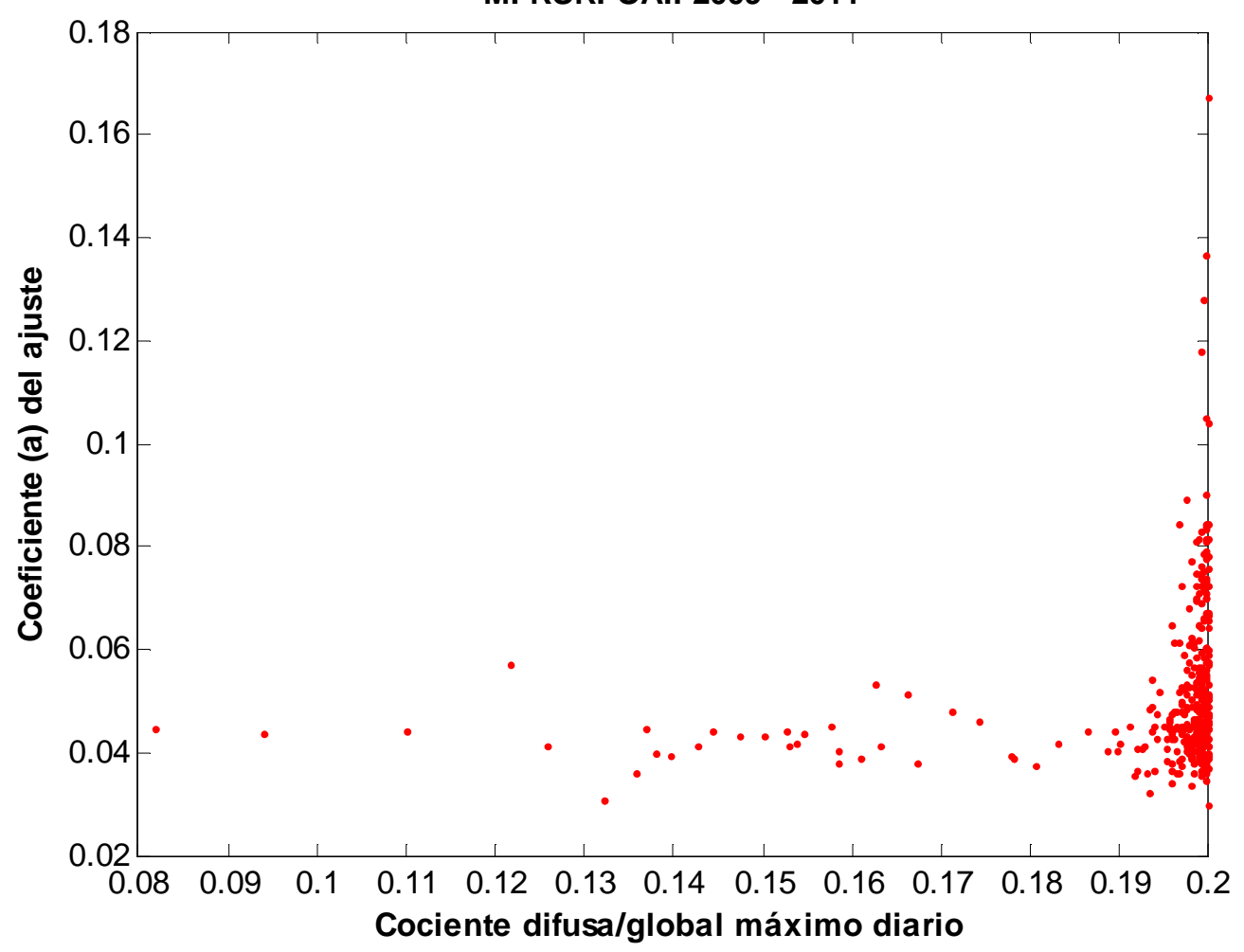

Cociente difusa/global diario y exponente de su ajuste a una función potencial simple MFRSR. OAI. 2009 - 2011

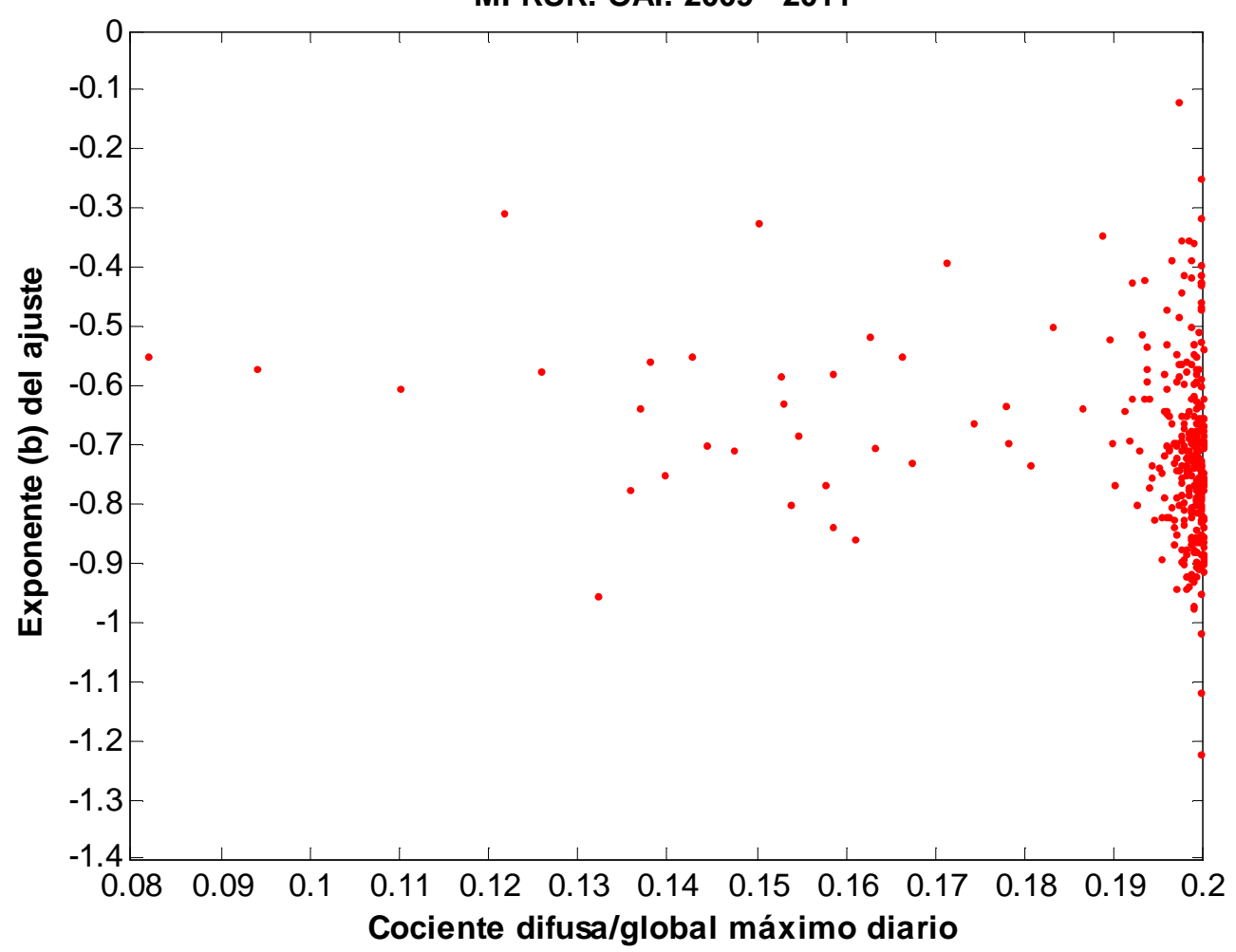

Figura 2.3.1. Parámetros asociados al cociente difusa/global del MFRSR y su relación con el valor máximo diario de este para los días utilizados en el proceso de calibración del canal de banda ancha del MFRSR en el período 2009-2011. Arriba, el coeficiente, y abajo, el exponente del ajuste a una función potencial simple. 


\subsection{Comparación radiación BSRN y MFRSR}

Para finalizar este capítulo, veamos cuáles son las diferencias entre las series de radiación integrada diaria global, difusa y directa normal de la BSRN y las del radiómetro MFRSR tras la calibración.

Estas series se han obtenido integrando numéricamente por la regla del trapecio entre orto y ocaso de cada día todos aquellos datos válidos, estuviesen 0 no afectados por nubes. Para el caso del radiómetro, se excluyeron aquellos datos cuya etiqueta o flag asociado era igual a -1, según los criterios que previamente ya habíamos establecido. Cada integración diaria llevaba implícito un error debido a la discretización o número de valores disponibles cada día, el cual variaba de un día para otro. La radiación diaria integrada se expresa en unidades de $M J /\left(m^{2} \times\right.$ día $)$ (Mega julios por $\mathrm{m}^{2}$ y día).

En la figura 2.4.1 se representa la radiancia global horizontal diaria de la BSRN frente a la del MFRSR para todos los días del período estudiado. El ajuste es razonablemente bueno con un alto coeficiente de determinación y un pequeño valor del error cuadrático medio. El coeficiente de conversión entre una serie y otra, dado por la pendiente de la recta de ajuste, es próximo a la unidad. El ajuste por mínimos cuadrados es simple, sin criterios de robustez.

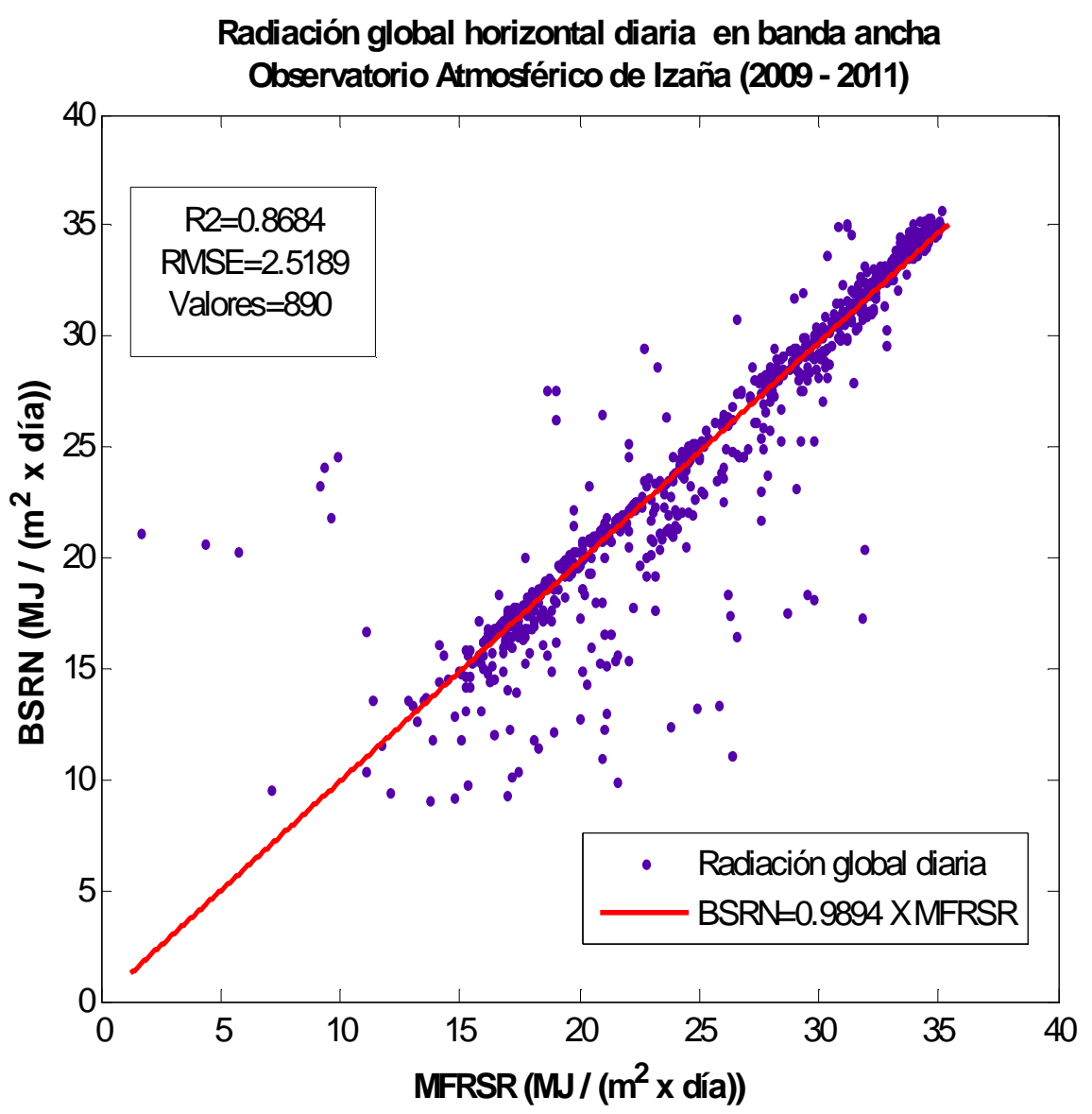

Figuras 2.4.1. Radiación global diaria horizontal en banda ancha de la BSRN y del MFRSR medida en el OAl en el período $2009-2011$. 
En el caso de la radiación difusa horizontal y directa normal tenemos ajustes similares pero de menor correlación cuyos parámetros serían los dados en la tabla 2.4.1.

\begin{tabular}{|c|c|c|c|c|c|c|c|}
\hline \multicolumn{4}{|c|}{ Difusa horizontal } & \multicolumn{4}{c|}{ Directa normal } \\
\hline R2 & RMSE & $\mathrm{N}$ & Coef. & R2 & RMSE & $\mathrm{N}$ & Coef. \\
\hline 0.6308 & 1.6962 & 854 & 0.8922 & 0.8330 & 4.1854 & 855 & 0.9977 \\
\hline
\end{tabular}

Tabla 2.4.1. Número de valores (N), error cuadrático medio (RMSE), coeficiente de determinación (R2) y pendiente (Coef) de los ajustes lineales para la radiancia difusa horizontal y directa normal medida por la BSRN y el MFRSR en el OAI en el período 2009-2011.

En las figuras 2.4 .2 se representan las componentes respectivas de la radiación diaria integrada para BSRN y MFRSR de manera conjunta.

Radiación global horizontal diaria en Izaña

2009-2011

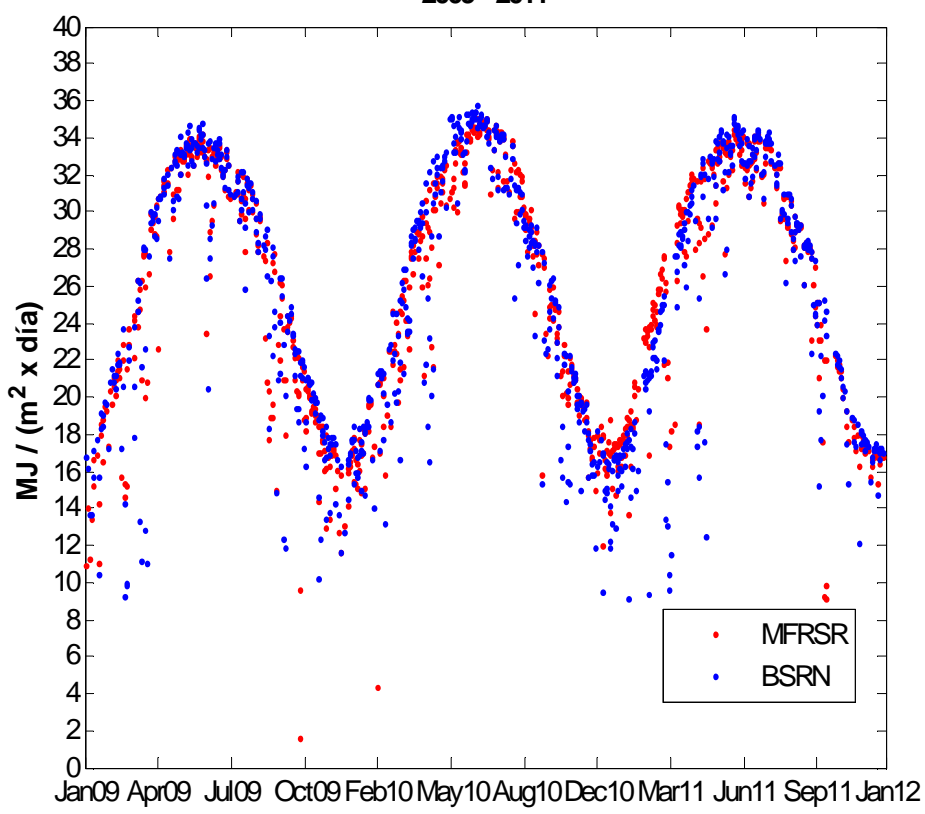

Fecha

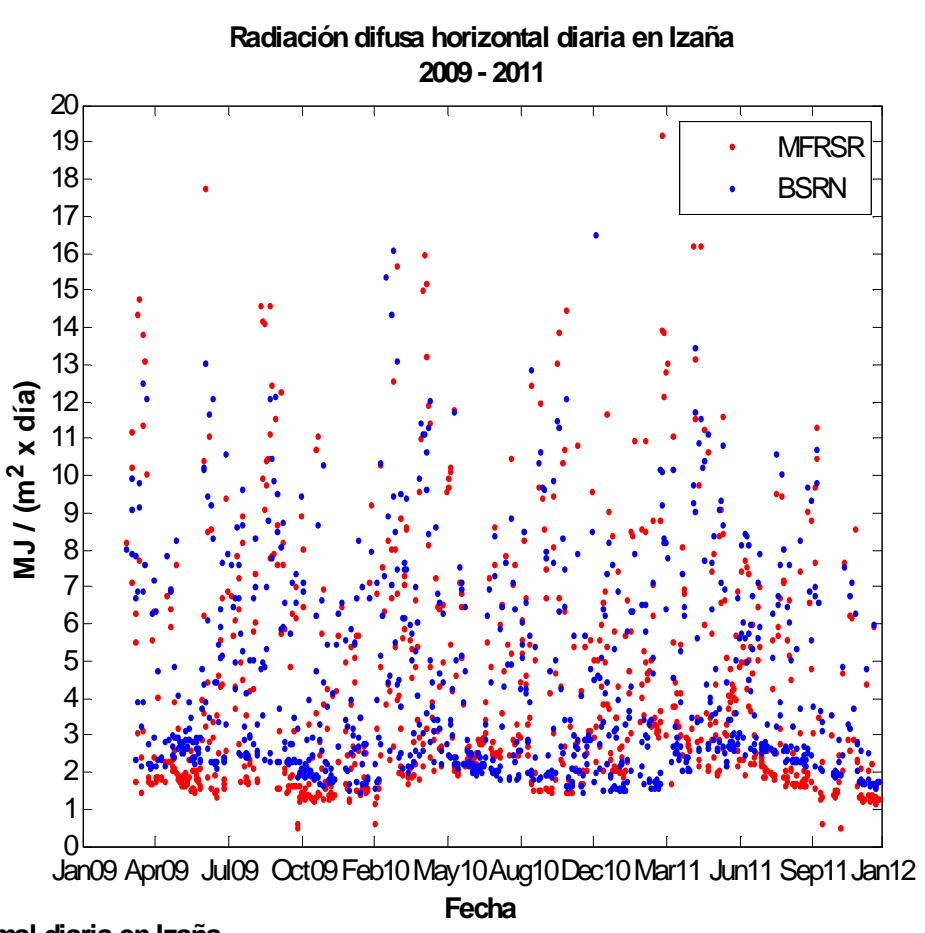

Radiación directa normal diaria en Izaña

2009 - 2011

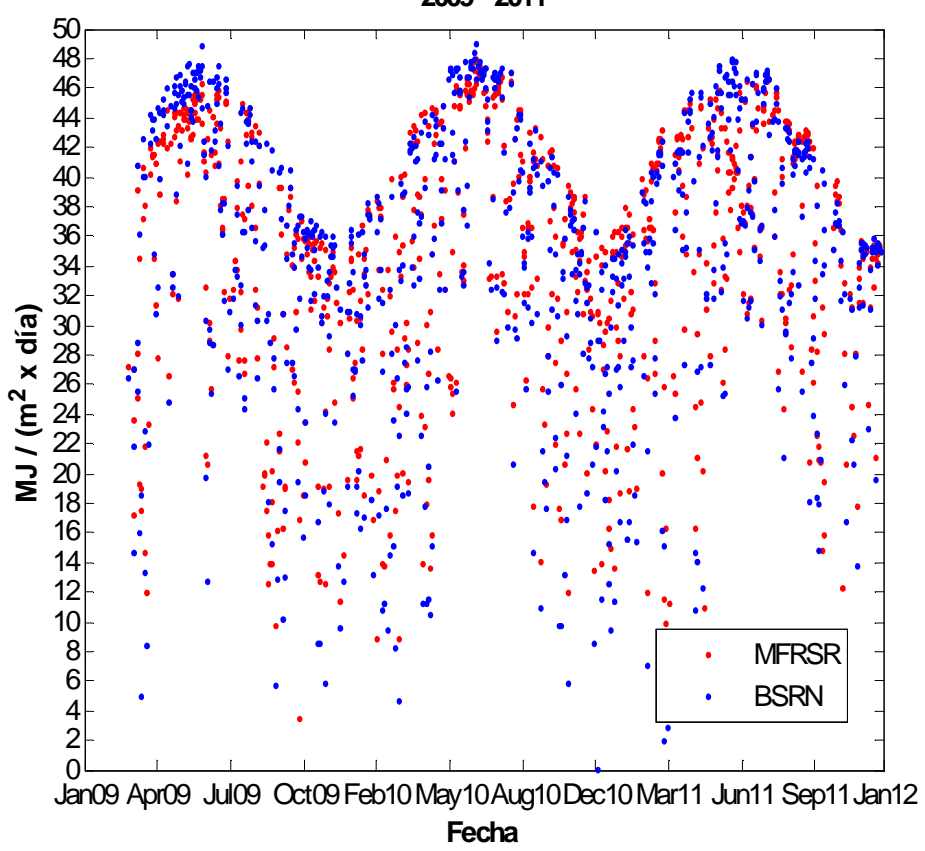


Figuras 2.4.2. Radiación global horizontal, difusa horizontal y directa normal del MFRSR y la BSRN. Izaña $2009-2011$.

Durante la mayor parte del año, las medidas de radiación solar en Izaña se distribuyen en el intervalo temporal comprendido entre las $8 \mathrm{~h}$ y las $18 \mathrm{~h}$ UTC. Podemos considerar entonces que tenemos una media de 10 horas diarias de datos minutales de radiación; suponiendo que la radiación que se mide cada minuto es igual a la que se mediría cada segundo dentro de dicho minuto, tendríamos una media diaria de 36000 segundos de medidas de radiación. De esta manera, podemos estimar de una manera fácil y razonable un valor representativo de la potencia media de radiación solar diaria multiplicando por $10^{6}$ el valor de la radiación integrada diaria en $M J /\left(m^{2} \times\right.$ día $)$ y dividiendo el resultado por 36000 . Así, $1 \mathrm{MJ} /\left(\mathrm{m}^{2} \times\right.$ día $)$ equivaldría a una irradiancia media diaria de $27.78 \mathrm{~W} / \mathrm{m}^{2}$.

Ahora bien, la incertidumbre en una medida se define como la dispersión de los valores que podrían razonablemente ser atribuidos o asignados a la propia medida. El objetivo marcado en 2004 para la incertidumbre máxima permitida en las medidas realizadas con instrumentación BSRN es del $2 \%$ para el total diario en el caso de la radiación global y difusa, y del $0.5 \%$ del total diario en el caso de la radiación directa normal. Teniendo en cuenta que los máximos diarios integrados de la BSRN en nuestra serie se sitúan en torno a los 35 $M J /\left(m^{2} \times\right.$ día $)$ para la radiación global, un $2 \%$ de esta cantidad sería equivalente a $0.7 \mathrm{MJ} /\left(\mathrm{m}^{2} \times\right.$ día $)$. En el caso de la radiación directa normal, los máximos diarios de la serie se encuentran en torno a $47 \mathrm{MJ} /\left(\mathrm{m}^{2} \times\right.$ día $)$; un $0.5 \%$ de esta cantidad serían $0.24 \mathrm{MJ} /\left(\mathrm{m}^{2} \times\right.$ día $)$. Para la difusa, los máximos diarios pueden alcanzar incluso los $20 \mathrm{MJ} /\left(\mathrm{m}^{2} \times\right.$ día $)$. Un $2 \%$ de esta cantidad serían 0.4 $M J /\left(m^{2} \times\right.$ dia $) \cdot$

El $67 \%$ de las diferencias en valor absoluto de la radiación global horizontal diaria medida por el MFRSR y la BSRN están dentro del error instrumental de esta. Esta cantidad se reduce al $26 \%$ en el caso de la radiación difusa y al $11 \%$ para el caso de la radiación directa normal. Podríamos concluir que MFRSR y BSRN son razonablemente equivalentes para la medida de la radiación global horizontal, pero no así, para la componente directa normal y difusa horizontal.

Esto puede explicarse debido a que la irradiancia global horizontal en el MFRSR se obtiene directamente a través de su sensor y, por tanto, no es calculada; sin embargo, los valores que el MFRSR obtiene para la irradiancia difusa horizontal y la directa normal no son directos sino que son calculados (por tanto, se obtienen de forma indirecta) a partir de otras medidas a las que aplican una serie de correcciones y ajustes. Así, la difusa horizontal se obtiene restando a la medida de irradiancia a través del sensor, cuando la banda de 
sombra cubre el difusor, la contribución del exceso de sombra proyectada por esta debido a su propia geometría. Para ello se realizan cálculos utilizando dos medidas laterales con la banda a ambos lados del difusor. Por otro lado, la irradiancia directa normal se calcula por el radiómetro restando a la global horizontal la difusa horizontal corregida mediante el procedimiento anterior y dividiendo dicha diferencia por el coseno del ángulo cenital solar.

Estas limitaciones habrá que tenerlas en cuenta en la evaluación del forzamiento radiativo por lo que restringiremos nuestro estudio únicamente a la radiación global horizontal para aquellos días dentro del período 2009 - 2011 en los que las diferencias en valor absoluto entre MFRSR y BSRN caen dentro del error instrumental de la BSRN para esta componente. Esto lo veremos en el capítulo 5. 


\section{Descripción de los modelos radiativos}

En este capítulo, describiremos los modelos con los que hemos trabajado en el desarrollo de esta nota técnica. Los tres modelos son válidos únicamente para cielos despejados, y el primero de ellos, el modelo de Gueymard, sólo evalúa la componente directa normal de la radiación en banda ancha. El adjetivo "ampliado" se lo añadimos nosotros para indicar que, a partir de la salida del modelo para la componente directa normal y a partir de la modelización del cociente difusa/global, hemos estimado, a posteriori, las componentes global y difusa horizontales para una atmósfera real que hubiesen correspondido a la componente directa normal suministrada por el modelo. Abordaremos también la determinación de la ampliación de este modelo a las componentes global y difusa para el caso de una atmósfera ideal. Tanto el modelo $\mathrm{C}$ de lqbal como el modelo SOL-GOA dan como salida las tres componentes de la irradiancia. Sin embargo, el modelo $C$ de lqbal, sólo sirve para banda ancha, mientras que el modelo SOL-GOA puede ser usado también de forma espectral.

\subsection{Modelo de Gueymard "ampliado"}

\section{Irradiancia directa normal a la dirección de incidencia}

Un primer modelo que usaremos para simular la irradiación solar recibida, será el propuesto por Gueymard C. A., (1998), que es válido para irradiancia directa normal a la dirección de incidencia, en banda ancha, y para cielos despejados. El método, modelado físicamente, está basado en predicciones preliminares de irradiancia espectral para un gran rango de condiciones atmosféricas idealizadas. Tras realizar una integración espectral se obtiene una parametrización de la irradiancia directa en banda ancha usando las principales variables atmosféricas. En este método, se consideran hasta 7 procesos individuales de atenuación atmosférica que son: la dispersión Rayleigh, la absorción por ozono $\left(\mathrm{O}_{3}\right)$ y por dióxido de nitrógeno $\left(\mathrm{NO}_{2}\right)$ troposférico y estratosférico, la absorción de los gases uniformemente mezclados y la del vapor de agua y la extinción (fundamentalmente dispersión) por aerosoles.

Según Gueymard, por similitud con la ley de Bouguer-Lambert-Beer, la cual, estrictamente hablando, sólo es válida para la radiación monocromática, la transmitancia de la irradiancia directa en banda ancha puede expresarse de la siguiente manera:

$$
E_{b n} / E_{0 n}=\exp \left(-m_{R} \delta_{R}-m_{o} \delta_{o}-m_{n s} \delta_{n s}-m_{n t} \delta_{n t}-m_{g} \delta_{g}-m_{w} \delta_{w}-m_{a} \delta_{a}\right)
$$

Aquí $E_{b n}=\int_{0}^{\infty} E_{b n \lambda} d \lambda$ es la irradiancia directa en banda ancha obtenida como integración en todo el rango espectral de las irradiancias espectrales directas, $E_{b n \lambda}$ recibidas en un plano normal a la dirección de incidencia; 
$E_{0 n}=\int_{0}^{\infty} E_{0 n \lambda} d \lambda$ es la integración espectral de las irradiancias espectrales extraterrestres, $E_{0 n \lambda}$ a la distancia Tierra-Sol del día en cuestión, y finalmente, $m_{i} \delta_{i}$ representa la contribución a la atenuación de irradiancia directa en banda ancha del componente i-ésimo atmosférico expresada como producto de su masa óptica relativa, $m_{i}$ por su espesor óptico en banda ancha, $\delta_{i}$. Los subíndices $R, o, n s, n t, g, w$ y a hacen referencia a Rayleigh, ozono, $\mathrm{NO}_{2}$ estratosférico y troposférico, gases uniformemente mezclados, vapor de agua y aerosoles, respectivamente.

La cantidad $E_{b n}$ es medida y suministrada directamente por el radiómetro, y el valor promedio de $E_{0 n}$, denominado constante solar, se toma como 1288 $\frac{W}{m^{2}}$, ya que, vamos a particularizar el modelo para el rango espectral 330nm2600nm.

A continuación, exponemos las distintas parametrizaciones asociadas a las variables que intervienen en (3.1.1) deducidas por Gueymard.

Si llamamos $Z$ al ángulo cenital solar (은), tenemos que $\mu_{0}=\cos Z$ y las masas ópticas relativas de Rayleigh y del vapor de agua se toman, respectivamente, como:

$$
\begin{aligned}
& m_{R}=\left[\mu_{0}+0.45665 Z^{0.07}(96.4836-Z)^{-1.6970}\right]^{-1} \\
& m_{w}=\left[\mu_{0}+0.031141 Z^{0.1}(92.4710-Z)^{-1.3814}\right]^{-1}
\end{aligned}
$$

Por otro lado, como los perfiles de densidad vertical de los aerosoles, del vapor de agua y del $\mathrm{NO}_{2}$ troposférico son muy parecidos entre sí, y las masas ópticas son sensibles a dichos perfiles, podemos asumir de una manera simplificada y de forma aproximada que sus masas ópticas son iguales, es decir: $m_{w}=m_{n t}=m_{a}$

En una atmósfera completamente limpia, $\delta_{a}=0$ En una atmósfera completamente seca, $\delta_{w}=0$ Llamando $\delta_{c}$ al espesor óptico en banda ancha para una atmósfera limpia y seca, podremos expresarlo y aproximarlo como: $\delta_{c}=\delta_{R}+\delta_{o}+\delta_{n s}+\delta_{g}$

Este espesor óptico es parametrizado mediante funciones simples cuyos coeficientes se obtienen ajustando datos numéricos a través de la técnica de mínimos cuadrados. Definiendo $q=1-p / p_{0}$ donde $p_{0}=1013.25$ (hPa) y $p$ es la presión actual en la estación, el espesor óptico de banda ancha para una atmósfera limpia y seca se obtiene como: 


$$
\delta_{c}=f_{1}(p, m R) \times\left[f_{2}\left(u_{0}, m_{R}\right)+f_{3}\left(m_{R}\right)\right]+f_{4}\left(u_{0}, m_{R}\right)+f_{5}\left(u_{n}, m_{R}\right)
$$

Donde:

$$
\begin{gathered}
f_{1}=\left(a_{0}+a_{1} m_{R}\right) /\left(1+a_{2} m_{R}\right) \\
f_{2}=b_{0}+b_{1} m_{R}^{0.25}+b_{2} \ln \left(m_{R}\right) \\
f_{3}=\left(0.19758+0.00088585 m_{R}-0.097557 m_{R}^{0.2}\right) /\left(1+0.0044767 m_{R}\right) \\
f_{4}=\left(c_{0}+c_{1} m_{R}^{-0.72}\right) / \exp \left(1+c_{2} m_{R}\right) \\
f_{5}=u_{n s} \times\left\lfloor 2.8669-0.078633\left(\ln \left(m_{R}\right)\right)^{2.36}\right\rfloor
\end{gathered}
$$

Y siendo:

$$
\begin{gathered}
a_{0}=1-0.98173 q \\
a_{1}=0.18164-0.24259 q+0.050739 q^{2} \\
a_{2}=0.18164-0.17005 q-0.0084949 q^{2} \\
b_{0}=-0.0080617+0.028303 u_{0}-0.014055 u_{0}^{2} \\
b_{1}=0.011318-0.041018 u_{0}+0.023471 u_{0}^{2} \\
b_{2}=-0.0044577+0.016728 u_{0}-0.01091 u_{0}^{2} \\
c_{0}=0.0036916+0.047361 u_{0}+0.0058324 u_{0}^{2} \\
c_{1}=0.015471+0.061662 u_{0}-0.044022 u_{0}^{2} \\
c_{2}=0.039904-0.038633 u_{0}+0.054899 u_{0}^{2}
\end{gathered}
$$

Los valores de ozono en la columna atmosférica y de $\mathrm{NO}_{2}$ estratosférico son, respectivamente, $u_{0}$ y $u_{n s}$ Ambos han de venir expresados en $\mathrm{atm} \times \mathrm{cm}$.

De manera similar, el espesor óptico del vapor de agua en banda ancha se obtiene de la siguiente parametrización:

$$
\delta_{w}=M \times\left\lfloor g_{1}+g_{2} M m_{w}+g_{3}\left(M m_{w}\right)^{1.28}\right] /\left(1+g_{4} M m_{w}\right)
$$


Donde:

$$
\begin{aligned}
& M=\left(1.7135+0.10004 m_{w}+0.00053986 m_{w}^{2}\right) \\
& \left(1.7149+0.097294 m_{w}+0.002567 m_{w}^{2}\right) \\
& g_{1}=\left(\gamma_{1} w+\gamma_{2} w^{1.6}\right) /\left(1+\gamma_{3} w\right) \\
& g_{2}=\left(\varphi_{1} w+\varphi_{2} w^{1.6}\right) /\left(1+\varphi_{3} w\right) \\
& g_{3}=\left(\kappa_{1} w+\kappa_{2} w^{1.6}\right) /\left(1+\kappa_{3} w\right) \\
& g_{4}=\left(v_{1} w+v_{2} w^{0.62}\right) /\left(1+v_{3} w+v_{4} w^{2}\right) \\
& \gamma_{1}=(1.728-2.1451 q) /(1-0.96212 q) \\
& \gamma_{2}=(0.37042+0.64537 q) /(1+0.94528 q) \\
& \gamma_{3}=(3.5145-0.12483 q) /(1-0.34018 q) \\
& \varphi_{1}=(0.63889-0.81121 q) \times(1-0.79988 q) \\
& \varphi_{2}=(0.06836+0.49008 q) /(1+4.7234 q) \\
& \varphi_{3}=(2.1567+1.4546 q) /(1+0.038808 q) \\
& \kappa_{1}=(-0.1857+0.23871 q) /(1-0.84111 q) \\
& \kappa_{2}=(-0.022344-0.19312 q) /(1+6.2169 q) \\
& \kappa_{3}=(2.1709+1.6423 q) /(1+0.062545 q) \\
& v_{1}=3.3704+6.8096 q \\
& v_{2}=\left(12.487-18.517 q-0.4089 q^{2}\right) /(1-1.4104 q)
\end{aligned}
$$




$$
\begin{gathered}
v_{3}=\left(2.5024-0.56834 q-1.4623 q^{2}\right) /(1-1.0252 q) \\
v_{4}=\left(-0.030833-1.172 q-0.98878 q^{2}\right) /(1+31.546 q)
\end{gathered}
$$

$w=$ cantidad de agua precipitable en la columna atmosférica (cm.).

Por último, el espesor óptico del aerosol en banda ancha se obtiene como:

$$
\delta_{a}=\left(1 / m_{a}\right) \times\left[\ln \left(E_{0 n} / E_{b n}\right)-m_{R} \delta_{c}\right]-\delta_{w}-\delta_{n t}
$$

Donde:

$$
\delta_{n t}=u_{n t} \times\left[2.8669-0.078633\left(\ln \left(m_{a}\right)\right)^{2.36}\right]
$$

Y siendo $u_{n t}$ el contenido de $\mathrm{NO}_{2}$ troposférico expresado en $\mathrm{atm} \times \mathrm{cm}$

Se ha comprobado que, en la práctica, el espesor óptico del aerosol en banda ancha es aproximadamente igual al espesor óptico del aerosol en 550nm, o sea:

$$
\delta_{a}=e o a 550
$$

Como el modelo ajusta mejor así, esta será la expresión que usaremos. El espesor óptico en 550nm se puede obtener mediante la ley de Ångström a partir del de 500nm proporcionado por uno de los canales del PFR (Precision Filter Radiometer) ó Radiómetro de Filtros de Precisión:

$$
e o a 550=e 0 a 500 \times\left(\frac{0.550}{0.500}\right)^{-\alpha}
$$

Donde $\alpha$ es el exponente de Ångström.

Como valor $u_{0}$ de ozono en columna tomaremos un valor climatológico correspondiente a cada día de un año estándar de 366 días calculado en el período 1998-2009 a partir de las medidas diarias en el OAl de un espectrofotómetro Brewer. La climatología se obtuvo mediante una media móvil cíclica en períodos de 11 días centrados en el día en cuestión y aplicada a las medianas de los valores de ozono en columna que, para un mismo día, había en los diferentes años del período.

La cantidad de dióxido de nitrógeno en la columna atmosférica es proporcionada y modelizada por el INTA (Instituto Nacional de Tecnología 
Aeroespacial) a partir de las medidas del dispositivo DOAS (Espectroscopia Óptica de Absorción Diferencial). La modelización diaria de la evolución del dióxido de nitrógeno en columna se asume como lineal, exceptuando los crepúsculos donde la variación es brusca. Los límites diarios entre los cuales se asume la evolución lineal del contenido de dióxido de nitrógeno dependen de la estación o época del año. Para los meses de octubre a diciembre (otoño) es cuando hay menos cantidad de $\mathrm{NO}_{2}$ en columna y la evolución lineal diaria sería entre $1.5 \times 10^{15} \frac{\text { moléculas }}{\mathrm{cm}^{2}}$ y $2.5 \times 10^{15} \frac{\text { moléculas }}{\mathrm{cm}^{2}}$.

De mayo a junio es cuando más cantidad de $\mathrm{NO}_{2}$ en columna se detecta al nivel del Observatorio de Izaña. Los umbrales para la variación lineal diaria serían en este caso: $3.0 \times 10^{15} \frac{\text { moléculas }}{\mathrm{cm}^{2}}$ y $4.5 \times 10^{15} \frac{\text { moléculas }}{\mathrm{cm}^{2}}$. Para el resto del año, los límites lineales serían: $2.5 \times 10^{15} \frac{\text { moléculas }}{\mathrm{cm}^{2}}$ y $3.5 \times 10^{15} \frac{\text { moléculas }}{\mathrm{cm}^{2}}$.

También asumimos que, de todo el $\mathrm{NO}_{2}$ en columna, el $10 \%$ de dicho valor se corresponde con $\mathrm{NO}_{2}$ troposférico medido desde el nivel del OAI $(2 \mathrm{~km}$, aproximadamente) hasta la tropopausa (12km, aproximadamente); el $90 \%$ restante, correspondería a $\mathrm{NO}_{2}$ estratosférico.

La expresión final para la irradiancia directa en banda ancha normal a la dirección de incidencia quedaría así:

$$
E_{b n}=E_{0 n} \times \exp \left(-m_{R} \delta_{c}-m_{a}\left(\delta_{a}+\delta_{w}+\delta_{n t}\right)\right)
$$

Como ya mencionamos anteriormente, un parámetro importante que sintetiza la expresión anterior, y es útil para la modelización y la calibración, es el coeficiente de turbiedad de Linke $T_{L}$. Este coeficiente, se define como el número de atmósferas limpias y secas necesarias para igualar la atenuación, y por tanto la irradiancia recibida, a la de la atmósfera real. Según esta definición, se puede entonces compactar la expresión (3.1.7) como:

$$
E_{b n}=E_{0 n} \times \exp \left(-m_{R} \delta_{c} T_{L}\right)
$$

De la igualdad entre las expresiones (3.1.7) y (3.1.8) se obtiene que el coeficiente de turbiedad de Linke puede expresarse como:

$$
T_{L}=1+\left(m_{a} / m_{R}\right) \times\left(\delta_{w}+\delta_{n t}+\delta_{a}\right) / \delta_{c}
$$

Esta expresión será útil a la hora de calcular la irradiancia recibida en situaciones ideales de ausencia de vapor de agua o aerosoles mediante el modelo y así poder estimar el forzamiento radiativo debido a estos componentes. 
La componente horizontal de la irradiancia directa se obtendría multiplicando la componente normal por el coseno del ángulo cenital solar, o sea:

$$
R=E_{b n} \times \mu_{0}
$$

\section{Irradiancia global y difusa horizontales}

Una vez determinada y modelada la componente directa de la irradiancia, la determinación de las componentes global y difusa se puede realizar de muchas formas y son muchos los modelos propuestos por diferentes autores: LibRadTran, SOL-GOA, MODTRAN, etc. En cada uno de ellos, se hace una aproximación basada en diferentes hipótesis.

Nosotros, adoptaremos la formulación sencilla expuesta al final del capítulo 2 cuando se trató sobre la modelización del cociente difusa/global. Esto sería algo que no forma parte del modelo de Gueymard sino que es añadido por nosotros y es por lo que le llamamos Gueymard "ampliado".

La relación entre componentes de irradiancias global, difusa y directa horizontales dada por (2.1.2) se cumple siempre para cualquier modelo y para cualquier situación. Si dividimos ambos miembros de (2.1.2) por la irradiancia global y substituimos la expresión (2.1.3) del cociente difusa/global y la (3.1.10) de la irradiancia directa horizontal en la expresión resultante tenemos que:

$$
\begin{gathered}
G=D+R \\
1=\frac{D}{G}+\frac{R}{G}=I_{c}+\frac{R}{G} \\
G=\frac{R}{1-a \mu_{0}^{b}}=\frac{E_{b n} \mu_{0}}{1-a \mu_{0}^{b}}
\end{gathered}
$$

La obtención de la difusa horizontal es ya inmediata a partir de lo anterior y de la relación (2.1.3):

$$
D=I_{c} G=a \mu_{0}^{b} G=\frac{E_{b n} a \mu_{0}^{b+1}}{1-a \mu_{0}^{b}}
$$

Para evaluar las irradiancias difusa y global horizontales en una atmósfera ideal, nos basaremos en la expresión del factor de turbiedad de Linke dada por (3.1.9).

En situación de ausencia de aerosoles o de vapor de agua, el factor de turbiedad de Linke se transforma, respectivamente, en:

$$
T_{L a}=1+\left(m_{a} / m_{R}\right) \times\left(\delta_{w}+\delta_{n t}\right) / \delta_{c}
$$




$$
T_{L w}=1+\left(m_{a} / m_{R}\right) \times\left(\delta_{n t}+\delta_{a}\right) / \delta_{c}
$$

La irradiancia difusa horizontal la estimamos de forma análoga a como lo hace el modelo r.sun (http://re.jrc.ec.europa.eu/pvgis/solres/solmod3.htm\#clearsky radiation):

$$
D_{h}=G_{0} \times \operatorname{Tn}\left(T_{L}\right) \times F_{d}\left(h_{0}\right)
$$

Donde, $G_{0}$ es la irradiancia extraterrestre corregida por la excentricidad de la órbita terrestre, $\operatorname{Tn}\left(T_{L}\right)$ es la función de transmisión difusa en una superficie horizontal y $F_{d}\left(h_{0}\right)$ es la función de altitud solar difusa. Estas funciones se calculan de la siguiente manera:

$$
\begin{gathered}
\operatorname{Tn}\left(T_{L}\right)=-0.015843+0.030543 \times T_{L}+0.0003797 \times T_{L}^{2} \\
F_{d}\left(h_{0}\right)=A_{1}+A_{2} \times \sin \left(h_{0}\right)+A_{3} \times \sin ^{2}\left(h_{0}\right) \\
A_{1}^{\prime}=0.26463-0.061581 \times T_{L}+0.0031408 \times T_{L}^{2} \\
A_{1}=\frac{0.0022}{\operatorname{Tn}\left(T_{L}\right)} \text { si } A_{1}^{\prime} \times \operatorname{Tn}\left(T_{L}\right)<0.0022 \\
A_{1}=A_{1}^{\prime} \operatorname{si~} A_{1}^{\prime} \times \operatorname{Tn}\left(T_{L}\right) \geq 0.0022 \\
A_{2}=2.04020+0.018945 \times T_{L}-0.011161 \times T_{L}^{2} \\
A_{3}=-1.3025+0.039231 \times T_{L}+0.0085079 \times T_{L}^{2}
\end{gathered}
$$

El factor de turbiedad de Linke $T_{L}$ se substituye por $T_{L a}$ o por $T_{L w}$ según tratemos con una atmósfera sin aerosoles 0 sin vapor de agua, respectivamente.

Finalmente, la irradiancia global horizontal para una atmósfera sin aerosoles o sin vapor de agua se obtiene mediante:

$$
G_{h}=D_{h}+R_{h}
$$

Donde $R_{h}$ es la irradiancia directa horizontal obtenida de la fórmula (3.1.10) substituyendo en $E_{b n}, \delta_{a}=0$, en ausencia de aerosoles, ó $\delta_{w}=0$, en ausencia de vapor de agua, según corresponda.

Todo lo visto hasta aquí es estrictamente válido para aquellos valores de irradiancias que no están afectados por nubes. El efecto de la nube en la irradiancia medida en un instante dado se evaluará restándole, al valor de la irradiancia real medida en dicho instante por nuestro equipo, el valor de la 
irradiancia simulada con el modelo tomando como valores de entrada al mismo los del instante en cuestión.

Según Gueymard, el error instrumental y la determinación precisa de la cantidad de agua precipitable son los principales factores que limitan la precisión del método y del modelo propuesto para la irradiancia directa normal. Por tanto, también serán los que más afecten a los valores de irradiancia global y difusa deducidos por nosotros de la forma que acabamos de exponer. Haremos una estimación general de los errores en el siguiente capítulo.

\subsection{Modelo $\mathrm{C}$ de Iqbal}

Este es un modelo basado en la comparación que Bird y Hulstrom realizaron entre varios modelos de insolación directa y el modelo riguroso SOLTRAN que se construyó a partir del LOWTRAN. Ellos, parametrizaron las transmitancias de los constituyentes atmosféricos tal y como se expondrá a continuación.

\section{Irradiancia directa normal a la dirección de incidencia}

Se obtiene como producto de las transmitancias de Rayleigh, ozono, gases uniformemente mezclados, vapor de agua y aerosoles.

$$
I_{n}=I_{0} \times \tau_{R} \times \tau_{o} \times \tau_{g} \times \tau_{w} \times \tau_{a}
$$

La transmitancia de Rayleigh se obtiene como:

$$
\tau_{R}=\exp \left(-0.0903 \times m_{a}^{0.84} \times\left(1.0+m_{a}-m_{a}^{1.01}\right)\right)
$$

Donde $m_{a}=\frac{m_{r} \times p}{1013.25}$, siendo $m_{r}=\frac{1}{\mu_{0}+0.15 \times(93.885-Z)^{-1.253}}$ la masa óptica relativa, $p$ es la presión en $\mathrm{hPa}$ de la estación, $\mu_{0}=\cos (Z)$ y $Z$ es el ángulo cenital solar en grados.

La transmitancia del ozono viene dada por:

$$
\begin{aligned}
& \tau_{o}=1-\left[0.1611 \times l \times m_{r} \times\left(1.0+193.48 \times l \times m_{r}\right)^{-0.3035}\right. \\
& \left.-0.002715 \times l \times m_{r} \times\left(1.0+0.044 \times l \times m_{r}+0.0003 \times\left(l \times m_{r}\right)^{2}\right)^{-1}\right]
\end{aligned}
$$

$l$ es el espesor óptico en columna del ozono expresado en cm.

La transmitancia para los gases uniformemente mezclados viene dada por:

$$
\tau_{g}=\exp \left(-0.0127 \times m_{a}^{0.26}\right)
$$

La transmitancia para el vapor de agua es: 


$$
\tau_{w}=1-2.4959 \times U_{1} \times\left[\left(1.0+79.034 \times U_{1}\right)^{0.6828}+6.385 \times U_{1}\right]^{-1}
$$

Siendo $U_{1}=w \times m_{r}=w^{\prime} \times\left(\frac{p}{1013.25}\right)^{3 / 4} \times\left(\frac{273}{T}\right)^{1 / 2}$ donde $w^{\prime}(p, T)$ es el agua precipitable en columna a la presión $p$ y temperatura $T$ expresada en $\mathrm{cm}$. y $w\left(p_{0}, T_{0}\right)$ es el agua precipitable en columna a la presión $p_{0}=1013.25 h P a$ y temperatura $T_{0}=273 \mathrm{~K}$ expresada en $\mathrm{cm}$. La temperatura $T$ de entrada al modelo será tomada como: $T=283 k$ ya que una temperatura de $10 \stackrel{\circ}{ } \mathrm{C}$ es un valor medio anual estadístico adecuado para Izaña.

La transmitancia del aerosol viene dada por:

$$
\tau_{a}=\exp \left[-k_{a}^{0.873} \times\left(1.0+k_{a}-k_{a}^{0.7088}\right) \times m_{a}^{0.9108}\right]
$$

Donde $k_{a}=0.2758 \times$ eoa $380+0.35 \times$ eoa 500 , siendo eoa380 y eoa500 los espesores ópticos o atenuación del aerosol a $380 \mathrm{~nm}$ y 500nm, respectivamente. Estos espesores ópticos se calculan mediante la ley potencial de Ångström.

\section{Irradiancia difusa horizontal}

La irradiancia difusa por dispersión Rayleigh, tras el primer paso a través de la atmósfera, se calcula mediante:

$$
I_{d r}=0.79 \times I_{0} \times \operatorname{exc} \times \mu_{0} \times \tau_{0} \times \tau_{g} \times \tau_{w} \times \tau_{a a} \times 0.5 \times \frac{1-\tau_{r}}{1-m_{a}+m_{a}^{1.02}}
$$

Donde $\tau_{a a}=1-\left(1-w_{0}\right) \times\left(1-m_{a}+m_{a}^{1.06}\right) \times\left(1-\tau_{a}\right)$ es la transmitancia de la irradiancia directa debida a la absorción del aerosol. El albedo de dispersión simple es $w_{0}$, recomendándose para él, un valor de 0.9 .

La irradiancia difusa por dispersión del aerosol, tras el primer paso por la atmósfera, viene dada por:

$$
I_{d a}=0.79 \times I_{0} \times \operatorname{exc} \times \mu_{0} \times \tau_{0} \times \tau_{g} \times \tau_{w} \times \tau_{a a} \times F_{c} \times \frac{1-\tau_{a} / \tau_{a a}}{1-m_{a}+m_{a}^{1.02}}
$$

Aquí, $F_{c}$ es la energía dispersada hacia delante dividida por la total dispersada. Se recomienda un valor de 0.84 .

La irradiancia difusa debida a múltiples reflexiones entre tierra y atmósfera, se obtiene así: 


$$
I_{d m}=\left(I_{n} \times \mu_{0}+I_{d r}+I_{d a}\right) \times \frac{\rho_{g} \times \rho_{a}}{1-\rho_{g} \times \rho_{a}}
$$

Donde $\rho_{g}$ es el albedo terrestre y $\rho_{a}$ es el albedo atmosférico para cielos despejados. El valor que tomaremos para el albedo terrestre es de 0.1. El albedo atmosférico viene dado por la expresión:

$$
\rho_{a}=0.0685+\left(1-F_{c}\right) \times\left(1-\frac{\tau_{a}}{\tau_{a a}}\right)
$$

Finalmente, la irradiancia difusa horizontal queda como:

$$
I_{d f}=I_{d r}+I_{d a}+I_{d m}
$$

\section{Irradiancia global horizontal}

Sería la suma de la directa horizontal y la difusa horizontal:

$$
I_{g}=I_{n} \times \mu_{0}+I_{d f}
$$

\subsection{Modelo SOL-GOA}

Este modelo fue desarrollado por el Grupo de Óptica Atmosférica de la Universidad de Valladolid (Cachorro et al., 2000; Cachorro et al., 2002) y está basado en la solución de la ecuación de transferencia radiativa mediante el método de Ambartsumian (Sobolev, 1963).

El modelo considera, por un lado, la interacción entre el scattering o dispersión de moléculas y aerosoles, y por otro, como efecto independiente, la absorción.

Es un modelo monocapa que admite como variables de entrada el parámetro de asimetría, la columna total de ozono, el albedo de dispersión simple y el albedo de superficie, el agua precipitable o vapor de agua en columna, la presión de la estación y los parámetros de aerosoles, o bien, directamente, el espesor óptico de aerosoles. (Cachorro et al., 1987; 1997).

\section{Irradiancia directa normal a la dirección de incidencia}

Viene dada por la ley de Bouguer-Lambert-Beer:

$$
F(\lambda)=F_{o}(\lambda) \exp (-\tau(\lambda) m)
$$

Donde $F(\lambda)$ es la irradiancia en el lugar de medida, $F_{0}(\lambda)$ es la irradiancia en la parte superior de la atmósfera, $m$ es la masa óptica y $\tau(\lambda)$ es el espesor óptico total de la atmósfera. Este espesor óptico total es la suma de los 
espesores ópticos debido a la dispersión por moléculas (Rayleigh), $\tau_{R}(\lambda)$, el cual debe corregirse por el factor $\frac{P}{P_{0}}$, donde $P$ es la presión del lugar y $P_{0}$ la presión al nivel del mar, el espesor óptico de aerosoles, $\tau_{a}(\lambda)$, y el debido a la absorción de los diferentes gases, $\tau_{g}(\lambda)$

$$
\tau(\lambda)=\tau_{R}(\lambda)+\tau_{a}(\lambda)+\tau_{g}(\lambda)
$$

El efecto de la suma de espesores ópticos también se puede expresar como producto de transmitancias debido a cada efecto independientemente:

$$
T(\lambda)=T_{R}(\lambda) T_{a}(\lambda) T_{g}(\lambda)
$$

El espesor óptico de Rayleigh debido a la dispersión por moléculas viene dado por la siguiente expresión:

$$
\tau_{R}(\lambda)=\frac{1}{117.2594 \lambda^{4}-1.3215 \lambda^{2}+0.00032-0.000076 \lambda^{-4}}
$$

El espesor óptico de aerosoles se obtiene de la ley de Ångström:

$$
\tau_{a}(\lambda)=\beta \lambda^{-\alpha}
$$

El espesor óptico del ozono se calcula mediante:

$$
\tau_{o 3}(\lambda)=C_{o 3}(\lambda) l m
$$

Donde $C_{o 3}(\lambda)$ son los coeficientes de absorción del ozono en $\mathrm{cm}^{-1}$ y $l$ es el contenido de ozono en Unidades Dobson.

\section{Transmitancia para la irradiancia global}

La transmitancia total para la irradiancia global viene dada por el producto de las transmitancias del efecto de dispersión de las moléculas (scattering Rayleigh), $T_{R}$, y la de aerosoles, $T_{a}$, según las expresiones (Sobolev,1963; Vermote and Tanré, 1992)):

$$
\begin{gathered}
T_{R}=\left(\frac{2}{3}+\frac{1}{m}\right)+\left(\frac{2}{3}-\frac{1}{m}\right) \frac{\exp \left(-\tau_{R} m\right)}{4 / 3+\tau_{R}} \\
T_{a}(\mu)=\frac{\left(1-r^{2}\right) \exp \left(-k \tau_{a} m\right)}{1-r_{o}^{2} \exp \left(k \tau_{a} m\right)}
\end{gathered}
$$


Donde $r_{0}=\frac{k-1+w_{0}}{k+1-w_{0}}$ y $k=\sqrt{\left(1-w_{0}\right)\left(1-w_{0} g\right)} \quad w_{0} \neq 1$

Siendo $g$ el parámetro de asimetría que toma valores entre -1 y 1 , indicando que la radiación es dispersada totalmente hacia atrás (valor -1) ó hacia delante (valor 1). Los valores usuales de $g$ varían entre 0.6 y 0.8 para las distribuciones de tamaños de los diferentes tipos de aerosoles en la atmósfera. La multiplicación de las dos expresiones anteriores presupone que no hay interacción entre esos dos procesos de dispersión.

Por último, la irradiancia difusa horizontal se calcula mediante diferencia entre la irradiancia global y la irradiancia directa horizontal, obtenida esta última multiplicando la irradiancia directa normal por el coseno del ángulo cenital.

\subsection{Variables de entrada a los modelos}

\section{Espesor óptico de aerosoles (EOA) en 550nm y parámetros de Ångström}

En los ficheros diarios de nivel 3 proporcionados por el Radiómetro de Filtros de Precisión (PFR) figuran valores de espesores ópticos minutales en las longitudes de onda de $862 \mathrm{~nm}, 500 \mathrm{~nm}, 412 \mathrm{~nm}$ y $368 \mathrm{~nm}$ así como valores minutales de los parámetros $\alpha$ y $\beta$ de Ångström. A partir del espesor óptico de 500nm y de la ley de Ångström (3.3.5) se obtiene el espesor óptico en 550nm como:

$$
e 0 a 550=e 0 a 500 \times\left(\frac{0.550}{0.500}\right)^{-\alpha}
$$

El espesor óptico en 500nm y los parámetros de Ångström correspondientes a cada medida del MFRSR se obtienen por interpolación ó extrapolación lineal entre los de los instantes PFR y MFRSR correspondientes a datos no nubosos. Cuando no es posible realizar estas interpolaciones ó extrapolaciones lineales se escogen valores por defecto. Para el espesor óptico en 500nm tomamos un valor de 0.02, y para los parámetros de Ångström, valores climatológicos.

\section{Agua precipitable o vapor de agua en columna}

Los valores de agua precipitable se calculan a partir de los retrasos de la señal GPS en la dirección cenital cuando pasa a través de la atmósfera. Se eligen retrasos cenitales totales de órbitas precisas GPS con resolución temporal horaria. Cuando no es posible, se utilizan órbitas ultrarrápidas con resolución temporal de 15 minutos. El dato asignado a cada medida MFRSR se obtiene por interpolación lineal del dato de agua precipitable GPS. Si un resultado concreto de la interpolación queda indeterminado, o no puede calcularse, se substituye por la mediana del resto de datos diarios interpolados de agua precipitable GPS. Si no disponemos de datos de órbitas GPS para un día concreto, elegimos el valor de agua precipitable obtenido a partir de los 
sondeos PTU. Este valor es la integral numérica desde los 2370 m.s.n.m hasta los 12000 m.s.n.m. del agua precipitable en columna. Si tampoco se tiene el dato del sondeo entonces se toma por defecto un dato climatológico diario de agua precipitable basado en la estadística para un año estándar de 366 días obtenida a partir de los sondeos diarios en el período (1994-2011).

\section{Ozono en columna}

El valor usado es un valor climatológico diario basado en la estadística para un año estándar de 366 días obtenida a partir de los valores diarios de ozono en la columna sobre Izaña para el período (1998-2009) calculados con un espectrofotómetro Brewer.

\section{Dióxido de nitrógeno en columna}

Tal y como se comentó previamente en la sección 3.1 se asume un modelo lineal entre los crepúsculos para la evolución del $\mathrm{NO}_{2}$ en columna.

\section{Albedo terrestre}

Se toma constante e igual a 0.1 .

Albedo de dispersión simple y parámetro de asimetría

Se toman constantes e iguales a 0.9 y 0.85 , respectivamente.

Presión atmosférica

Se toma constante e igual a $770 \mathrm{hPa}$.

Energía dispersada hacia delante dividida por la total dispersada

Se toma constante e igual a 0.84 . 


\section{Comparativa de los modelos usados}

Estudiamos aquí cuáles son las diferencias entre los valores reales medidos con el radiómetro calibrado y los simulados con cada uno de los modelos propuestos usando, como variables de entrada para cada día concreto, las descritas en el capítulo anterior. Únicamente, analizaremos tales diferencias para la irradiancia global horizontal (valores instantáneos en $\mathrm{W} / \mathrm{m}^{2}$ ), y lo haremos sólo, en aquellos días de nuestro período de estudio en los que la integral diaria de la irradiancia medida con nuestro radiómetro calibrado MFRSR se encuentra dentro del error instrumental de la obtenida con la BSRN. Como los modelos sólo sirven para días despejados, en la comparación usaremos únicamente datos etiquetados como no nubosos, según los criterios ya explicados en el capítulo 2. Para que los resultados obtenidos sean lo más generales posibles abarcaremos todos los ángulos cenitales entre orto y ocaso.

Presentamos en las figuras $4.1,4.2$ y 4.3 las salidas de irradiancias de los modelos Gueymard "ampliado", Iqbal y SOL-GOA, respectivamente, frente a las medidas reales de irradiancias del MFRSR calibrado para los días anteriormente seleccionados.

Irradiancia global horizontal en el OAl. (2009 - 2011)

Dias en los que abs(integral(MFRSR)-integral(BSRN) $)<0.7 \mathrm{MJ} /\left(\mathrm{m}^{2} \times\right.$ día)

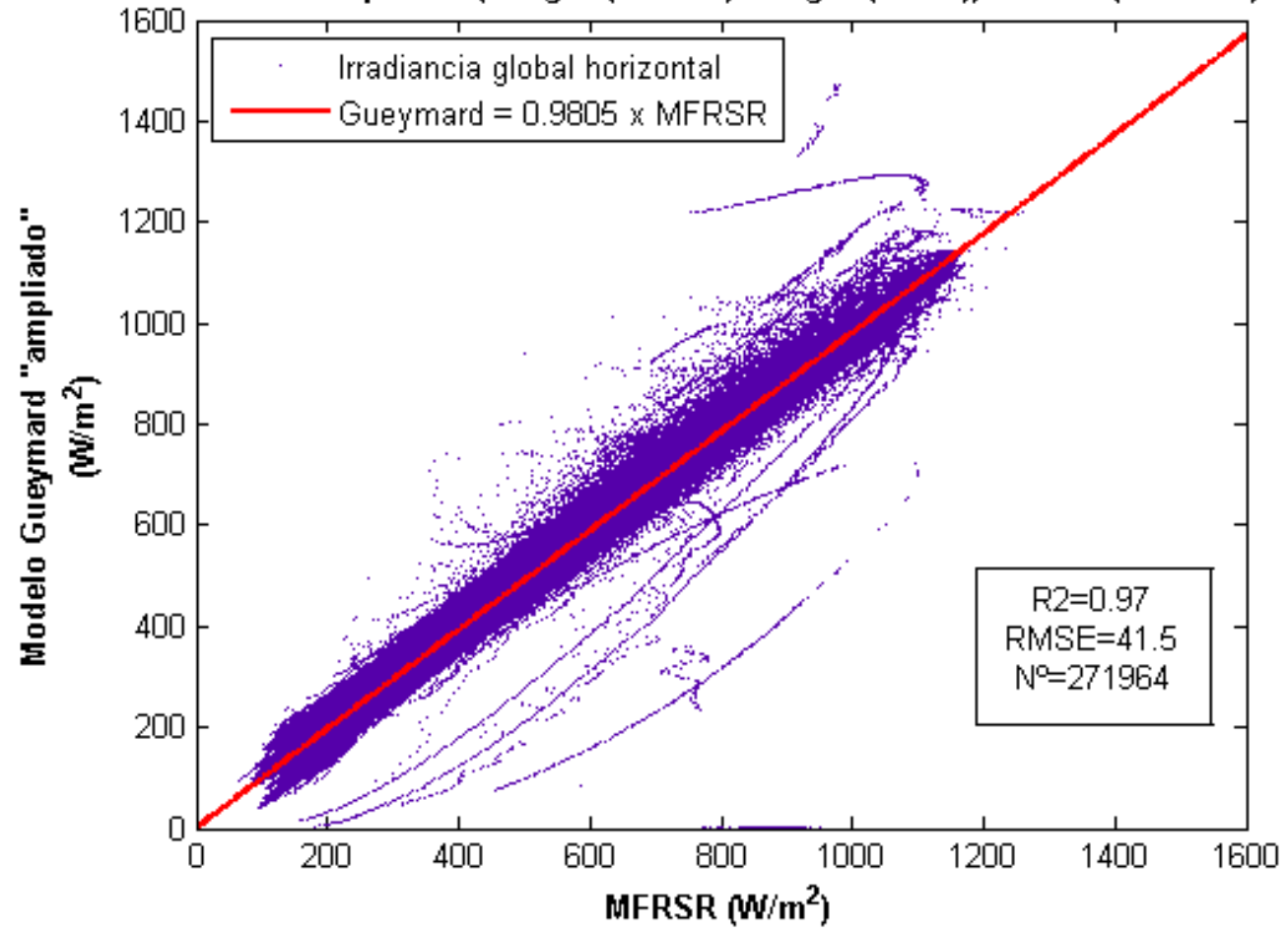

Figura 4.1. Ajustes para la irradiancia global horizontal entre las salidas del modelo de Gueymard "ampliado" y las medidas MFRSR para todos los ángulos cenitales entre orto y ocaso de datos sin nubes de aquellos días en los que las medidas MFRSR y BSRN se encuentran dentro del error instrumental de esta última. OAI (2009 - 2011). R2 es el coeficiente de determinación del ajuste; RMSE es el error cuadrático medio y $\mathrm{N}$ es el número de valores usados en las comparaciones. En la leyenda se especifica la ecuación de la recta de ajuste. 
Irradiancia global horizontal en el OAl. (2009 - 2011)

Días en los que abs(integral(MFRSR)-integral(BSRN) $<0.7 \mathrm{MJ} /\left(\mathrm{m}^{2} \mathrm{x}\right.$ día)

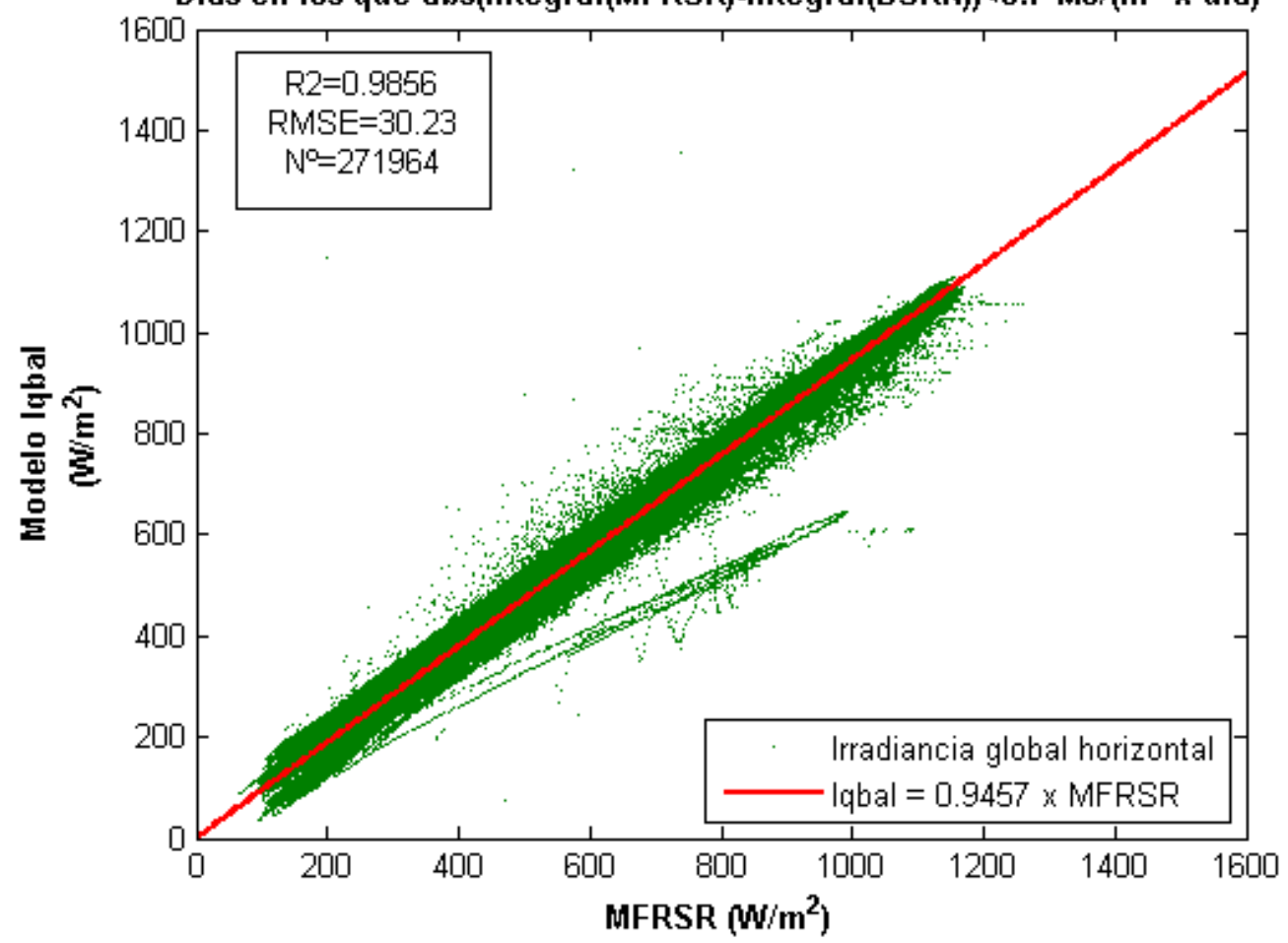

Figura 4.2. Ajustes para la irradiancia global horizontal entre las salidas del modelo de lqbal y las medidas MFRSR para todos los ángulos cenitales entre orto y ocaso de datos sin nubes de aquellos días en los que las medidas MFRSR y BSRN se encuentran dentro del error instrumental de esta última. OAI (2009 - 2011). R2 es el coeficiente de determinación del ajuste; RMSE es el error cuadrático medio y $\mathrm{N}$ es el número de valores usados en las comparaciones.

En la leyenda se especifica la ecuación de la recta de ajuste.

Irradiancia global horizontal en el OAl. (2009 - 2011)

Días en los que abs(integral(MFRSR)-integral(BSRN) $<0.7 \mathrm{MJ} /\left(\mathrm{m}^{2} \mathrm{x}\right.$ día)

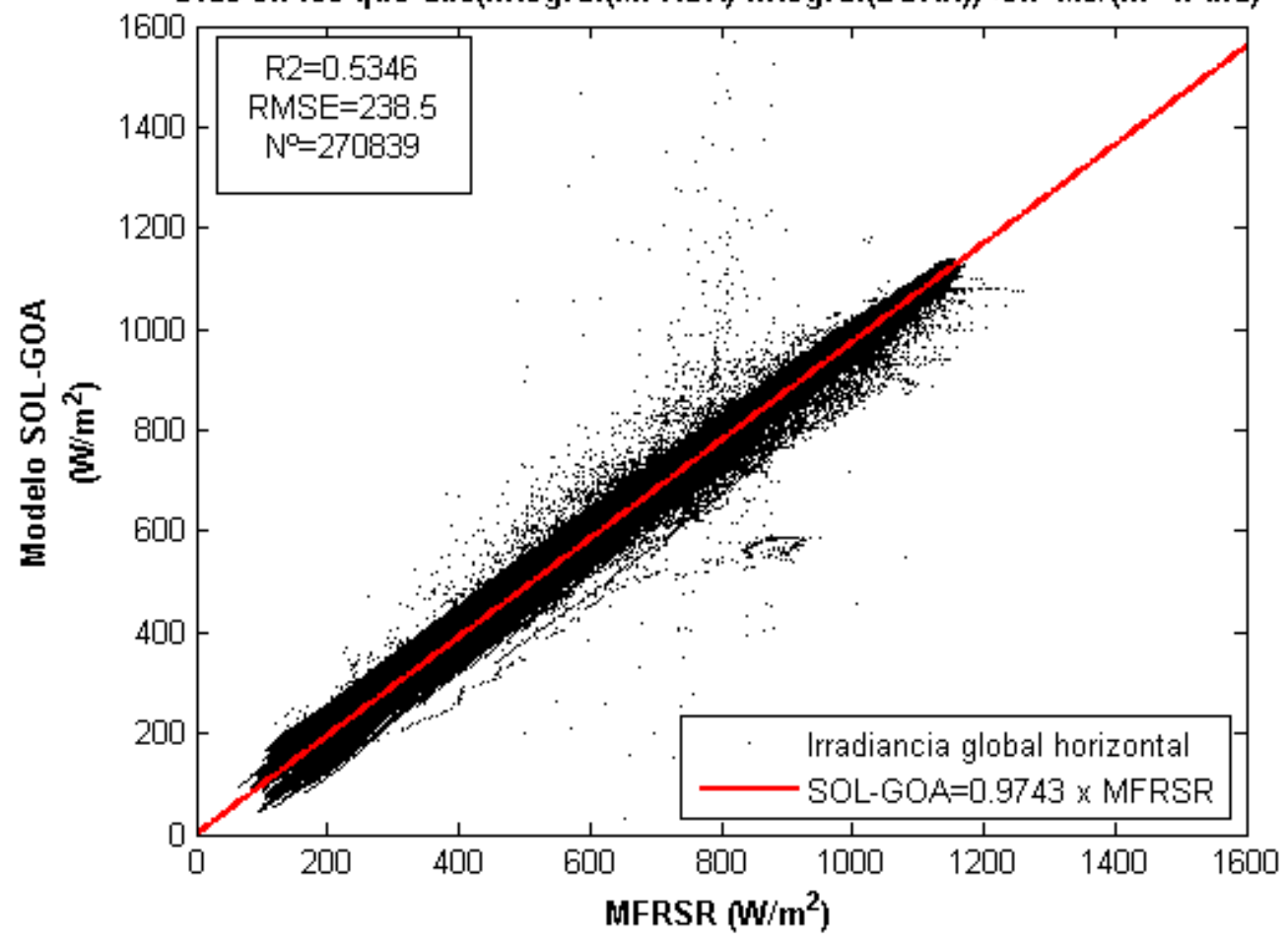


Figura 4.3. Ajustes para la irradiancia global horizontal entre las salidas del modelo SOLGOA y las medidas MFRSR para todos los ángulos cenitales entre orto y ocaso de datos sin nubes de aquellos días en los que las medidas MFRSR y BSRN se encuentran dentro del error instrumental de esta última. OAl (2009 - 2011). R2 es el coeficiente de determinación del ajuste; RMSE es el error cuadrático medio y $\mathrm{N}$ es el número de valores usados en las comparaciones. En la leyenda se especifica la ecuación de la recta de ajuste.

En cada una de las gráficas anteriores podemos ver los parámetros asociados con cada uno de los ajustes. Hay que indicar que estos ajustes se han realizado a rectas que pasen por el origen y se han hecho por la técnica de mínimos cuadrados sin aplicar ningún tipo de criterio de robustez.

Estos resultados nos muestran que, en general, los tres modelos ajustan bastante bien a las medidas y tienen buena correlación con ellas. Las correlaciones son bastante altas para los modelos de Gueymard "ampliado" e lqbal; en el caso del modelo SOL-GOA la correlación no es tan alta, como en los dos anteriores, aunque podemos considerar que da un valor aceptable. Por otra parte, este último modelo es el que más dispersión respecto del ajuste presenta de los tres. En este sentido, es el modelo de lqbal el menos disperso ofreciendo el menor de los errores cuadráticos medios. En realidad, y tal y como se aprecia en los parámetros de ajuste, los modelos de Gueymard "ampliado" e lqbal se comportan, en lo que a correlaciones y dispersión se refiere, de manera similar y diferente al modelo SOL-GOA. Por último, y en cuanto al coeficiente de conversión entre modelos y medidas MFRSR, decir que están todos próximos a la unidad pero por debajo de ella, si bien, el de Gueymard "ampliado" se aproxima más que los otros. Esto significa que las salidas de los tres modelos son, en promedio, más bajas que la medida real obtenida con el radiómetro MFRSR. Así, el modelo de lqbal infravalora la medida instrumental en un $6 \%$ aproximadamente, mientras que el SOL-GOA lo hace en un 3\% y el Gueymard "ampliado" infravalora en un $2 \%$, aproximadamente.

En las gráficas anteriores, vemos que, para los tres modelos, hay valores instantáneos puntuales que se alejan bastante de la zona del ajuste. Pensamos que esto podría ser debido, a que el equipo no ha medido correctamente en esos instantes de tiempo y/ó el algoritmo de detección de nubes haya dado una asignación errónea de etiqueta nubosa a dichos valores considerándolos como no afectados por nubes en vez de nubosos o inservibles, en su caso. No vamos a entrar a estudiar el por qué ha ocurrido esto, ya que, en cualquier caso, dichos valores puntuales son minoritarios comparados con el resto y no influyen, o lo hacen muy débilmente, en la integral diaria de la irradiancia del MFRSR, de forma que, las medidas de este y las de la BSRN, se mantienen dentro del error instrumental de esta última para los días seleccionados y representados. Hemos comprobado también que si hubiéramos realizado ajustes robustos, descartando aquellos valores erróneos y más alejados de los mismos, las correlaciones serían bastante altas en los tres modelos (mayores de 0.99) pero los coeficientes de las rectas de regresión apenas variarían de la situación actual.

Como la gran mayoría de los valores instantáneos en los días seleccionados correlacionan bastante bien con los tres modelos, las 
correlaciones y los resultados podemos considerarlos estadísticamente aceptables.

A la luz de estos resultados, asumimos el hecho de que el modelo empírico de Gueymard, en su versión "extendida" o "ampliada" a las componentes global y difusa horizontales propuesta por nosotros, es el que, en promedio, más parece ajustarse a las medidas calibradas del instrumento MFRSR para todos los ángulos cenitales ofreciendo, además, una alta correlación y una baja dispersión respecto del ajuste.

Por tanto, trabajaremos con este modelo a la hora de obtener y analizar los forzamientos radiativos del aerosol, vapor de agua y nubes en la radiación global horizontal, lo que será objeto del próximo capítulo. 


\section{Forzamiento del aerosol, vapor de agua y nubes}

Se entiende por forzamiento radiativo de un cierto componente atmosférico la diferencia entre la irradiancia real medida por un instrumento en un cierto rango espectral y la que hubiese medido el mismo instrumento en ausencia de dicho componente y que es simulada mediante un modelo.

Por todo lo dicho en el capítulo 4 sobre la comparativa de los modelos propuestos, el más conveniente de los tres es el modelo de Gueymard ampliado por nosotros. Este será el que usaremos a fin de obtener la evaluación de los forzamientos radiativos para el aerosol, el vapor de agua y las nubes con la mayor precisión que nos es posible. Las componentes difusa y global horizontales para la atmósfera ideal sin aerosoles o sin vapor de agua, necesarias para la evaluación del forzamiento radiativo, se han obtenido para este modelo como se indica en el capítulo 3 desde las fórmulas (3.1.14) a la (3.1.23).

El forzamiento radiativo de un componente se puede evaluar de forma instantánea, o bien, para todo un día, integrando entre orto y ocaso la irradiancia real recibida y la salida del modelo en ausencia del componente. Luego, se hace la diferencia entre ambas integrales. Pero el estudio del forzamiento instantáneo no tiene demasiada utilidad práctica si lo que queremos es evaluar el calentamiento o enfriamiento de la atmósfera. Por ello, estudiaremos el forzamiento total diario en el período comprendido entre 2009 y 2011.

Sin embargo, dado el análisis comparativo que se hizo en el capítulo 2 entre la radiación medida por la BSRN y la medida por el MFRSR, restringiremos nuestro estudio del forzamiento radiativo únicamente a la componente global horizontal de la irradiancia para aquellos días del período 2009 - 2011 donde la integral diaria obtenida sobre esta componente con el MFRSR y la obtenida con la BSRN difieren en valor absoluto como máximo en $0.7 \mathrm{MJ} /\left(\mathrm{m}^{2} \times\right.$ día $)$ que, como vimos en dicho capítulo, era el error máximo instrumental permitido.

A continuación, pasamos a describir cómo se ha obtenido este forzamiento radiativo.

Si el dato no es nuboso, el forzamiento radiativo debido a la presencia de aerosoles se obtendrá restando al valor real integrado diario de la irradiancia correspondiente, el valor integrado diario de la irradiancia simulada con el modelo y obtenida para una atmósfera sin aerosoles. Análogamente, el forzamiento radiativo debido a la presencia del vapor de agua se obtendrá restando al valor real integrado diario de la irradiancia correspondiente, el valor integrado diario de la irradiancia simulada con el modelo y obtenida para una atmósfera sin vapor de agua. Lógicamente, para estos datos, el forzamiento radiativo nuboso será 0 .

Cuando el dato esté afectado por nubes, antes de proceder a evaluar el forzamiento radiativo debido al vapor de agua o a los aerosoles, debemos restar la contribución debida al forzamiento causado por las propias nubes. El 
criterio que usaremos para ello será el de aproximar dicho dato con el del modelo usado (incluyendo aerosoles y vapor de agua), ya que, dicho modelo (al igual que los otros estudiados en esta nota técnica), es válido para días despejados, y por tanto, estima el valor de radiación en ausencia de nubes para las mismas condiciones en las que se está midiendo. El forzamiento radiativo nuboso se calculará como la diferencia entre el valor real integrado diario de la irradiancia correspondiente y el valor integrado diario de la irradiancia en la que los datos nubosos se han substituido por los del modelo. Una vez extraída esta contribución al forzamiento debida a la presencia de nubes, procederemos como si el dato fuera no nuboso y evaluaremos los forzamientos del aerosol y del vapor de agua en la forma explicada anteriormente. Evidentemente, todo esto comporta un cierto error puesto que el modelo no reproduce exactamente el valor real medido. Por ello, también evaluamos las diferencias entre la integración diaria de los datos reales y la de los datos simulados con el modelo para los mismos instantes de tiempo. Estas diferencias nos ayudarán a acotar la validez de los resultados obtenidos.

Suponiendo que la diferencia entre la salida del modelo para una atmósfera real y la medida del equipo sea la misma que entre la salida del modelo para una atmósfera ideal y la supuesta medida del equipo en dicha atmósfera, el error en la estimación del forzamiento radiativo vendría dado, en primera aproximación, por la diferencia entre modelo y equipo en la atmósfera real. Los errores por discretización en la integración diaria tanto de las medidas reales como de las simuladas juegan un papel secundario y menos importante. De hecho, el $90 \%$ de los valores absolutos de los errores de integración por discretización en las medidas reales están por debajo de $0.1 \mathrm{MJ} /\left(\mathrm{m}^{2} \mathrm{x}\right.$ día).

El promedio del valor absoluto de las diferencias entre medidas y modelo para los días seleccionados en los que las medidas MFRSR y BSRN están dentro del error instrumental de esta última es de $0.63 \mathrm{MJ} /\left(\mathrm{m}^{2} \mathrm{x}\right.$ día) y su desviación estándar es de $0.80 \mathrm{MJ} /\left(\mathrm{m}^{2} \mathrm{x}\right.$ día). Haciendo una estimación a "grosso" modo, podríamos decir entonces que el error en el cálculo del forzamiento radiativo en la radiación global está comprendido entre -0.17 y 1.43 $\mathrm{MJ} /\left(\mathrm{m}^{2} \mathrm{x}\right.$ día$)$.

A fin de hacernos una idea del orden de magnitud que representa tanto el forzamiento radiativo estimado como el error cometido en su evaluación, es muy conveniente disponer de los valores umbrales o límites entre los cuales oscila la serie de radiación diaria global. Ya en el capítulo 2 se presentaron las correspondientes series del MFRSR y de la BSRN para su comparación.

En ellas, se observaban unos ciclos de máximos y mínimos correspondientes a los meses de verano e invierno, respectivamente.

La serie de radiación global integrada diaria arroja unos mínimos anuales en invierno que están, sobre los $15^{M J} /\left(\mathrm{m}^{2} \times\right.$ día $)$, mientras que, los máximos anuales se sitúan en verano sobre los $35 M J /\left(m^{2} \times d i a\right)$. Los límites aludidos 
corresponden a días despejados o suficientemente despejados tanto en invierno como en verano.

Por tanto, el error estimado en el forzamiento radiativo representa entre el $0.5 \%$ y el $4 \%$, aproximadamente, del valor máximo de radiación global horizontal diaria (35 $\mathrm{MJ} /\left(\mathrm{m}^{2} \mathrm{x}\right.$ día)).

En la gráfica 5.1 representamos el forzamiento radiativo total diario en la radiación global horizontal para aerosoles, vapor de agua y nubes obtenido con el modelo de Gueymard "ampliado" para los días seleccionados dentro del período $2009-2011$.

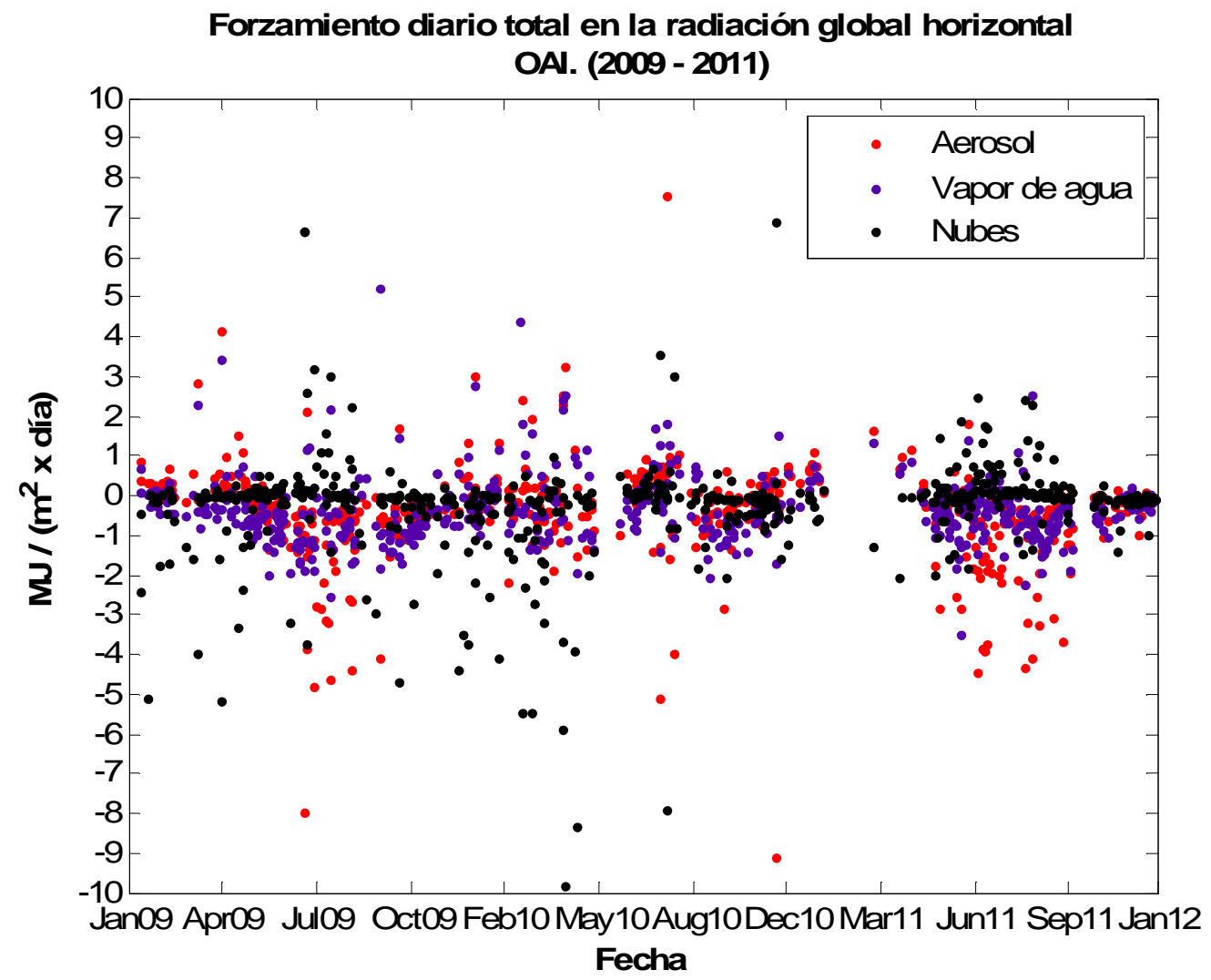

Figura 5.1. Forzamiento en la radiación global horizontal integrada diaria. En negro, el debido a la presencia de nubes y en azul y rojo el debido al vapor de agua y los aerosoles, respectivamente. MFRSR. Izaña (2009-2011).

En la figura anterior, pueden observarse unos forzamientos negativos mínimos, que tanto para aerosoles como para vapor de agua, se manifiestan en períodos de verano y otoño. Esto puede explicarse porque, en dichos períodos, es donde más cantidad de vapor de agua precipitable hay presente en la atmósfera, y por tanto, donde mayor será su influencia frente a una atmósfera que estuviese completamente seca. Por otro lado, también es en el período de verano donde más episodios de intrusión sahariana se dan sobre Izaña con presencia de gran cantidad de partículas aerosoles.

Un forzamiento radiativo negativo (tal y como se ha definido y calculado) se traduce en un enfriamiento de la atmósfera puesto que significa que habría 
más radiación en ausencia de un componente determinado que la que hay realmente con la presencia de dicho componente. $Y$ al revés, un forzamiento radiativo positivo, indicaría un calentamiento en la atmósfera debido a la influencia de la presencia del componente analizado, la cual se traduce en un aumento de la radiación respecto de la que habría sin la presencia de dicho componente.

El forzamiento nuboso observamos que también es muy variable. Su valor es 0 para días que son despejados o prácticamente despejados.

En la figura 5.2 se muestra una representación del forzamiento del aerosol frente al del vapor de agua en la radiación global horizontal para los días seleccionados. En ella, apreciamos más claramente en qué rango o límites nos movemos y nos permite expresar uno de ellos en función del otro.

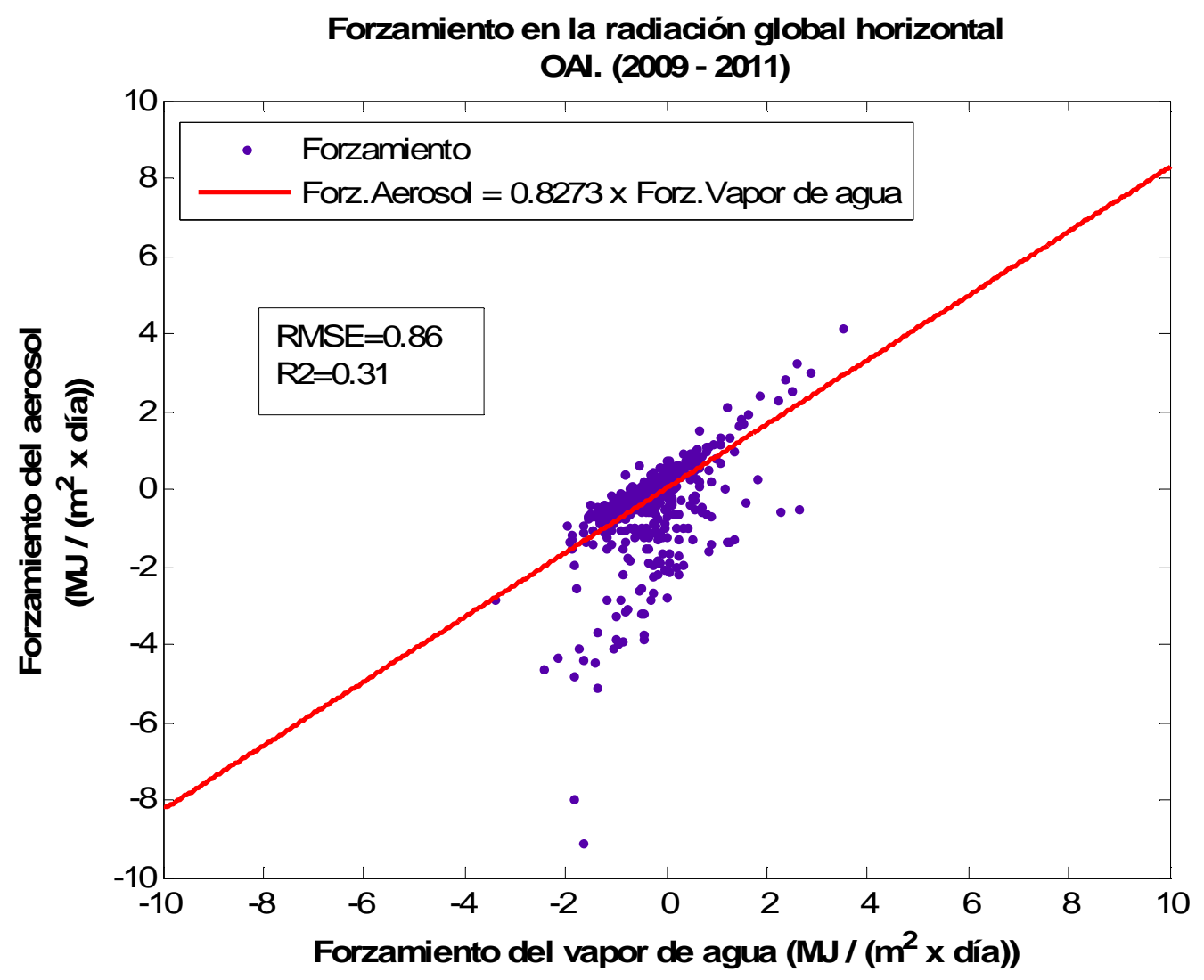

Figura 5.2. Forzamiento del aerosol frente al del vapor de agua para los días seleccionados. Izaña (2009 - 2011).

Parece que el forzamiento del aerosol, en términos generales, suele ser un $17 \%$ menor que el del vapor de agua. Sin embargo, la parte inferior bajo la recta de ajuste, nos indica la existencia de bastantes situaciones en las que el forzamiento del aerosol puede llegar a ser incluso el doble o el triple que el del vapor de agua. 
En la tabla 5.1, se muestran los valores estadísticos en cuartiles para el forzamiento en la componente global horizontal de la radiación en los días seleccionados. Los resultados vienen expresados en $M J /\left(m^{2} \times\right.$ día $)$.

\begin{tabular}{|c|c|c|c|}
\hline Forzamiento global horizontal & Aerosol & Vapor de agua & Nubes \\
\hline P25 & -0.60 & -0.82 & -0.23 \\
\hline P50 & -0.20 & -0.38 & 0.00 \\
\hline P75 & 0.11 & -0.01 & 0.13 \\
\hline Media & -0.34 & -0.59 & -0.24 \\
\hline
\end{tabular}

Tabla 5.1. Estadística básica en cuartiles para el forzamiento en la radiación global horizontal diaria debido a la presencia de aerosoles, vapor de agua y nubes. Izaña (2009 - 2011).

De la inspección de la tabla anterior, de las gráficas 5.1 y 5.2 , y de los errores estimados en el cálculo del forzamiento radiativo, observamos que estos se sitúan dentro del mismo orden de magnitud que los propios forzamientos. Este hecho puede estar ligado no sólo a la precisión de los modelos, sino también, a que las variables físicas más importantes que se utilizan como entradas a los mismos y que modulan sus salidas, presentan este mismo comportamiento. Por ejemplo, el espesor óptico de aerosoles (AOD, en inglés) en $500 \mathrm{~nm}$ en Izaña para días muy estables y limpios ("pristine days") suele alcanzar un valor máximo de 0.02 . Este valor es del mismo orden de magnitud que el error medio cometido en la evaluación del AOD por medio de los instrumentos más precisos (PFR), el cual se sitúa sobre 0.01. Algo análogo ocurre con el agua precipitable en columna determinada por GPS cuyo error se sitúa en torno a $1 \mathrm{~mm}$. En Izaña, tenemos atmósferas muy secas donde muchas veces el contenido de agua precipitable es de 2 ó $3 \mathrm{~mm}$ (el mismo orden de magnitud que el error). Además, en estos casos, estamos en el límite de detección de agua precipitable del GPS. Con esto, queremos indicar que también las variables de entrada al modelo tienen errores del mismo orden de magnitud que sus propios valores, lo cual, junto a los errores del propio modelo, se trasladará a las salidas obtenidas con este.

Cuando estudiamos en el capítulo 4 las diferencias entre las medidas reales del radiómetro y las simuladas por cada uno de los modelos propuestos, vimos que estas diferencias discrepaban entre sí de una manera más o menos marcada dependiendo de cuál fuera el modelo usado. Por tanto, esto debe transmitirse igualmente a los resultados obtenidos en el estudio del forzamiento radiativo, los cuales, también presentarán una discrepancia en función de la dependencia con el modelo usado que podría llegar a ser importante. Si hubiésemos empleado otro modelo distinto al aquí usado para evaluar el forzamiento, probablemente, hubiéramos obtenido resultados similares pero diferentes a los presentados en este trabajo.

Cuanto más se aproxime la simulación de un modelo radiativo al valor real de irradiancia medido por el instrumento calibrado, tanto más adecuado y conveniente para el estudio del forzamiento radiativo será el modelo. En nuestro caso, la elección del modelo de Gueymard ("ampliado") para la 
evaluación del forzamiento estuvo determinada por ser el que menos diferencias presentaba frente a las medidas reales y calibradas del MFRSR.

Seguidamente, compararemos los resultados obtenidos aquí con los calculados por otros autores para otras zonas del mundo, utilizando otro tipo de modelos convenientes. Los resultados, aunque no iguales, deberían ser similares o estar dentro del mismo orden de magnitud. No obstante, debemos tener en cuenta que el calentamiento o enfriamiento de la atmósfera no se presenta de manera uniforme para todos los ámbitos geográficos por lo que nuestros resultados sólo pueden ser considerados como locales y no necesariamente representativos de otros lugares.

Para hacer la comparación, vamos a calcular la eficiencia del forzamiento radiativo del aerosol como la variación de este por unidad de espesor óptico en $550 \mathrm{~nm}$. Vendrá dada, por tanto, por el cociente entre forzamiento y espesor óptico. La aplicaremos a la radiación global horizontal. Para ello, tomaremos una serie de días despejados dentro del período 2009 -2011 pero con una carga estable de aerosoles atmosféricos durante todo el día de forma que, el espesor óptico medio diario, tome valores significativos de turbiedad superando el umbral de 0.1 .

Muchos autores calculan el forzamiento radiativo diario expresándolo en W / $\mathrm{m}^{2} \mathrm{y}$ hora. Esto se obtiene dividiendo entre 24 la integral diaria de la irradiancia entre orto y ocaso expresada en $\mathrm{J} / \mathrm{m}^{2}$, con lo que en realidad tendríamos una especie de forzamiento horario medio al día, y luego dividiendo este forzamiento horario medio entre 3600 ( $\mathrm{n}^{\circ}$ de segundos en una hora) para expresarlo en $\mathrm{W} / \mathrm{m}^{2}$. En realidad, con este procedimiento, lo que hacemos es repartir uniformemente la radiación recibida durante las horas de sol entre las 24 horas diarias. Es una forma de expresarlo que está extendida en la literatura por lo que la asumiremos para ver si los resultados obtenidos son comparables con los de dichos autores (Bush and Valero, 2003).

Los forzamientos del aerosol expresados en $\mathrm{W} /\left(\mathrm{m}^{2} \mathrm{x}\right.$ hora) para tales días son los que se muestran en las figuras 5.3 y 5.4 .

Forzamiento promedio horario diario del aerosol en la irradiancia global horizontal Días despejados con valores estables de espesor óptico de aerosol superiores a 0.1

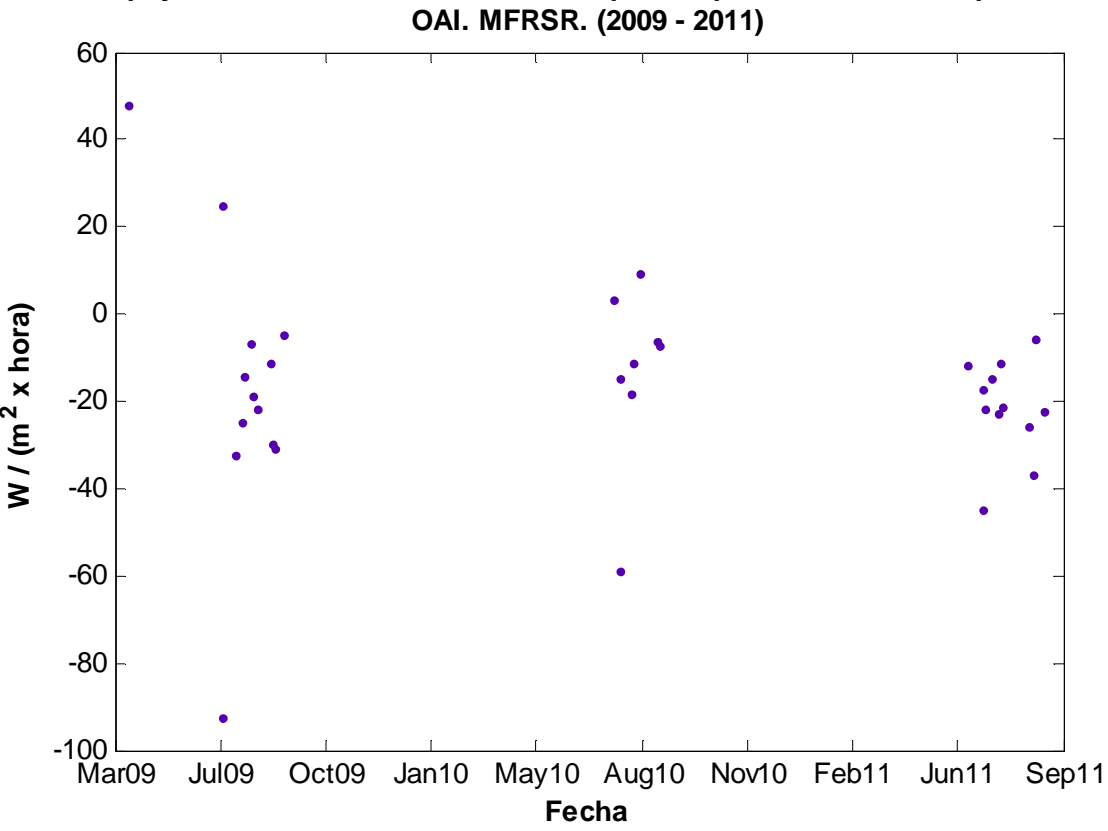


Figura 5.3. Forzamiento promedio horario diario del aerosol en la irradiancia global horizontal para días con espesor óptico estable superior a 0.1. Izaña $(2009-2011)$.

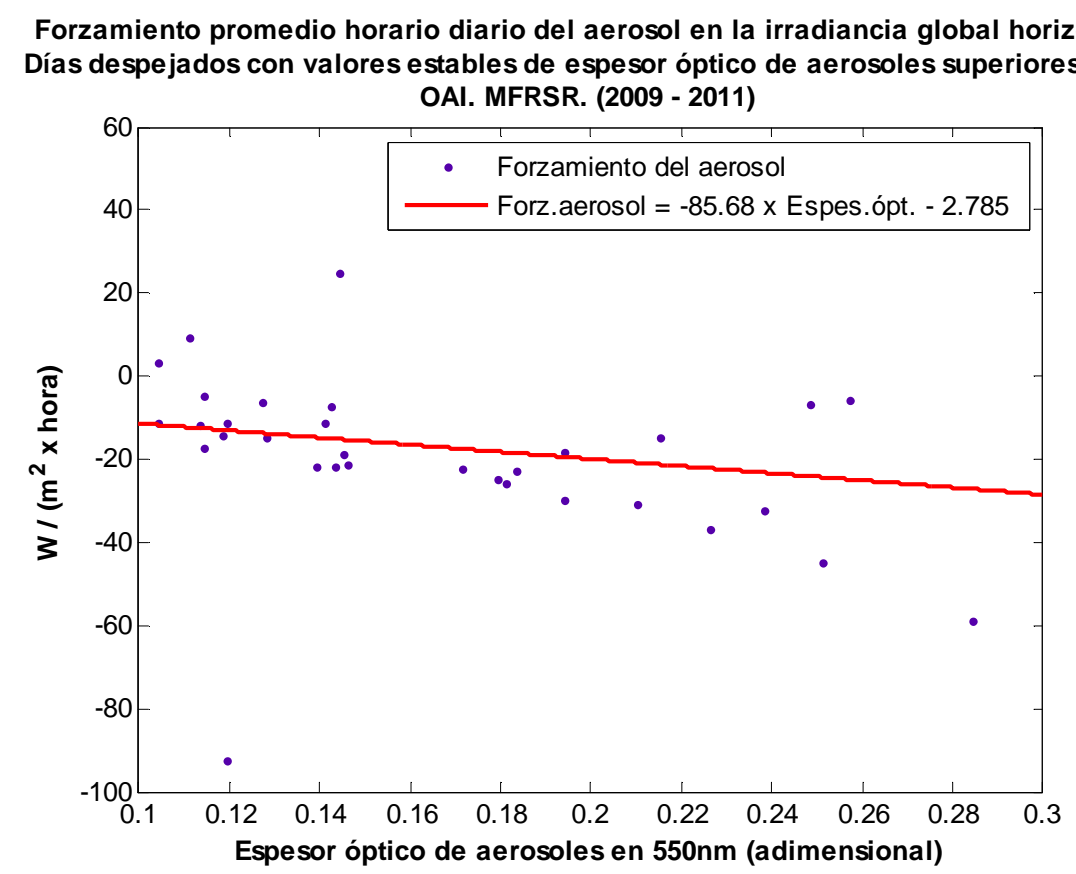

Figura 5.4. Forzamiento promedio horario diario del aerosol en la irradiancia global horizontal en función de su espesor óptico. Izaña (2009 - 2011).

El valor medio de la eficiencia del forzamiento del aerosol para los días considerados es de $-104.36\left(\mathrm{~W} /\left(\mathrm{m}^{2} \mathrm{x}\right.\right.$ hora $\left.\left.\mathrm{x} \tau\right)\right)$, donde $\tau$ es el espesor óptico de aerosoles en 550nm. Otros autores, definen la eficiencia del forzamiento como la pendiente del ajuste lineal entre el forzamiento radiativo y el espesor óptico de aerosoles (Rajev and Ramanathan, (2001)). En nuestro caso, hemos obtenido un valor de $-85.68\left(\mathrm{~W} /\left(\mathrm{m}^{2} \mathrm{x}\right.\right.$ hora $\left.\left.\mathrm{x} \tau\right)\right)$.

Autores como Valenzuela et al., (2012), reportan valores de eficiencia media diaria de aerosoles sobre la zona de Granada (España) para eventos de polvo sahariano entre 2005 y 2010 de -74 y $-65\left(\mathrm{~W} /\left(\mathrm{m}^{2} \mathrm{x}\right.\right.$ hora $\left.\left.\mathrm{x} \tau\right)\right)$. Ge, J. M. et al (2010) hablan de una eficiencia del forzamiento radiativo medio diario del aerosol de -95.1 (W / $\left(\mathrm{m}^{2} \mathrm{x}\right.$ hora $\left.\left.\mathrm{x} \tau\right)\right)$ sobre el noroeste de China en 2008. Bush and Valero, (2002) obtuvieron una eficiencia diaria de -73 (W/ $/ \mathrm{m}^{2} \mathrm{x}$ hora $\mathrm{x} \tau)$ ) en la campaña INDOEX (Océano Índico) en 1999. Todos estos resultados, aunque distintos, están en consonancia con los aquí deducidos.

El mismo cálculo y análisis podríamos hacer para la eficiencia del forzamiento del vapor de agua y el de las nubes, pero esto último es bastante más complicado y no queremos extendernos más en este trabajo.

Pensamos que hemos cubierto los objetivos propuestos en la introducción, ofreciendo, ante todo, una metodología de trabajo y dando unos resultados muy generales y orientativos. Dejamos para el futuro estudios más amplios que abarquen un período de tiempo más extenso y en el que usemos otro tipo de modelos, aún más precisos, que nos permitan abordar el estudio de tendencias y otras componentes del forzamiento radiativo a largo plazo. 


\section{Bibliografía}

1. Barnard J. C., Long C. N., (2004): A Simple Empirical Equation to Calculate Cloud Optical Thickness Using Shortwave Broadband Measurements. J. Appl. Meteor., 43, 1057 - 1066.

2. Bason F., (2008): Linke's Turbidity Factor Applied to Worldwide Global Horizontal Irradiance Measurements. EuroSun 2008, Lissabon, Portugal, October 2008.

3. Benas, N., Hatzianastassiou, N., Matsoukas, C., Fotiadi, A., Mihalopoulos, N., and Vardavas, I., (2011): Aerosol shortwave daily radiative effect and forcing based on MODIS Level 2 data in the Eastern Mediterranean (Crete), Atmos. Chem. Phys. Discuss., 11, 19881-19925, doi: 10.5194/acpd-11-19881-2011, 2011.

4. Bird R. E., Riordan C., (1986): Simple Solar Spectral Model for Direct and Diffuse Irradiance on Horizontal and Tilted Planes at the Earth's Surface for Cloudless Atmospheres. J. Climate Appl. Meteor., 25, 87 97.

5. Bush, B. C., and F. P. J. Valero. Spectral aerosol radiative forcing at the surface during the Indian Ocean Experiment (INDEOX), J. Geophys. Re., 107, 8003,10pp, doi:0.1029/2000JD000020, (2002).

6. Bush, B. C., and F. P. J. Valero. Surface aerosol radiative forcing at Gosan during the ACE-Asia campaign,J. Geophys. Res., 108, 8660, 8pp, doi:10.1029/2002JD003233, (2003).

7. Cachorro, V.E., De Frutos, A.M., and Casanova, J.L., (1987): The influence of Ångström parameters on calculated direct solar spectral irradiances at high turbidity, Solar Energy, 39, 399-407.

8. Cachorro, V.E., Utrillas, P., Martínez-Lozano, J.A., and De Frutos, A.M., (1997): A preliminary assessment between a detailed two stream shortwave narrow-band and spectral radiation measurements, Sol. Energy, 61, 265-273.

9. Cachorro, V.E., Vergaz, R. y de Frutos A.M., (2000): Características del modelo de radiación en el rango visible-cercano infrarrojo y ultravioleta UVA_GOA. Informe técnico del GOA-UVA al INM. Report interno (http://goa.uva.es).

10. Cachorro V.E., Vergaz, R., Sorribas, M. y de Frutos, A.M., (2002): Descripción y estudio de sensibilidad del modelo de radiación ultravioleta "UVA-GOA, $3^{\text {a }}$ Asamblea Hispano-Portuguesa de Geodesia y Geofísica. Proceeding Tomo II, 1219-23 (S13). Valencia 2002. ISBN: 84-9705-2994. 
11. De la Casinière A., Bokoye A. I., Cabot T., (1997): Direct Solar Spectral Irradiance Measurements and Updated Simple Transmittance Models. J. Appl. Meteor., 36, $509-520$.

12. García R. D., (2011): Aplicación de modelos de transferencia radiativa para el control operativo del programa BSRN (Baseline Surface Radiation Network) del Centro de Investigación Atmosférico de Izaña. Tesis Doctoral. Universidad de Valladolid. Diciembre de 2011.

13. Ge, J. M., J. Su, T. P. Ackerman, Q. Fu, J. P. Huang, and J. S. Shi., (2010): Dust aerosol optical properties retrieval and radiative forcing over northwestern China during the 2008 China-U.S. joint field experiment, J. Geophys. Res., 115, D00K12, doi: 10.1029/2009JD013263, [printed 116(D7), 2011].

14. Gueymard C. A., (1998): Turbidity Determination from Broadband Irradiance Measurements: A Detailed Multicoefficient Approach. J. Appl. Meteor., 37, $414-435$.

15. Hofierka J., Šúri M., (2002): The solar radiation model for Open source GIS: implementation and applications. Proceedings of the Open source GIS - GRASS users conference 2002 - Trento, Italy, 11 - 13 September 2002.

16. Iqbal M., (1983): An introduction to solar radiation. Academic Press Canada. 1983.

17. Ineichen, P., (2008): Conversion function between the Linke turbidity and the atmospheric water vapour and aerosol content. Sol. Energy (2008), doi: 10.1016/j.solener.2008.04.010.

18. Kasten F., (1980): A simple parameterization of the pyrheliometric formula for determining the Linke turbidity factor. Meteorol. Rdsch. 33, $124-127$ (August 1980).

19. Kim D., Ramanathan V., (2008): Solar radiation budget and radiative forcing due to aerosols and clouds. J. Geophys. Res., 113, D02203, doi: 10.1029/2007JD008434.

20. Kurucz, R., (1992): Synthetic infrared spectra, in Proceedings of the 154th Symposium of the International Astronomical Union (IAU). Tucson, Arizona, March 2-6, 1992, Kluwer, Acad., Norwell, MA, (1992).

21. Long C. N., Ackerman T. P., (2000): Identification of clear skies from broadband pyranometer measurements and calculation of downwelling shortwave cloud effects, J. Geophys. Res., 105(D12), 15,609-15,626, doi: 10.1029/2000JD900077.

22. McArthur L. J. B, (2005): Baseline Surface Radiation Network (BSRN). Operations Manual. Version 2.1. WMO/TD-No. 1274, WCRP-121. 
23. Rajev, K., and V. Ramanathan. Direct observations of clear-sky aerosol radiative forcing from space during the Indian Ocean Experiment, J. Geophys Res., 106, 17,221-17, 235, doi: 10.1029/2000JD900723, (2001).

24. Remund J., Wald L., Lefevre M., Ranchin T., Page J., (2003): Worldwide Linke turbidity information. Proceedings of ISES Solar World Congress, 16 - 19 June, Göteborg, Sweden, CD - ROM published by International Solar Energy Society.

25. Romero Campos P.M., Cuevas E., Ramos R., Valdés M., Schneider M., (2009): Programa de vapor de agua en columna del Centro de Investigación Atmosférica de Izaña: Análisis e Intercomparación de diferentes Técnicas de Medida. NTD CIAI-1. Agencia Estatal de Meteorología. Ministerio de Medio Ambiente, y Medio Rural y Marino. NIPO: 784-09-009-9.

26. Sobolev. (1963): A Treatise on Radiative Transfer. D. Van Nostrand Company, Inc.

27. Solar radiation model r.sun and its implementation in GRASS GIS. European Commission. Joint Research Center.

28. Tendeku F., (1995): Retrieval of Atmospheric Turbidity Coefficient and Water Column Density from Solar Irradiance Data. Proceedings Arkansas Academy of Science, Vol.49, 1995.

29. Valenzuela, A., F.J. Olmo, H. Lyamani, M. Antón, A. Quirantes and L. Alados-Arboledas. Aerosol radiative forcing during African desert dust events (2005-2010) over Southeastern Spain. Atmos. Chem. Phys., 12, 10331-10351, doi:10.5194/acp-12-10331-2012, (2012).

30. Vermote E.F. and Tanré, D., (1992): Analytical Expressions for Radiative Properties of Planar Rayleigh Scattering Media Including Polarization Contribution, Journal of Quantitative Spectroscopy and Radiative Transfer, 47, (4), 305-314. 